\title{
A new approach to deformation equations of noncommutative KP hierarchies*
}

\author{
Aristophanes Dimakis \\ Department of Financial and Management Engineering, \\ University of the Aegean, 31 Fostini Str., GR-82100 Chios, Greece \\ dimakis@aegean.gr \\ Folkert Müller-Hoissen \\ Max-Planck-Institute for Dynamics and Self-Organization \\ Bunsenstrasse 10, D-37073 Göttingen, Germany \\ folkert.mueller-hoissen@ds.mpg.de
}

\begin{abstract}
Partly inspired by Sato's theory of the Kadomtsev-Petviashvili (KP) hierarchy, we start with a quite general hierarchy of linear ordinary differential equations in a space of matrices and derive from it a matrix Riccati hierarchy. The latter is then shown to exhibit an underlying 'weakly nonassociative' (WNA) algebra structure, from which we can conclude, refering to previous work, that any solution of the Riccati system also solves the potential KP hierarchy (in the corresponding matrix algebra). We then turn to the case where the components of the matrices are multiplied using a (generalized) star product. Associated with the deformation parameters, there are additional symmetries (flow equations) which enlarge the respective KP hierarchy. They have a compact formulation in terms of the WNA structure. We also present a formulation of the KP hierarchy equations themselves as deformation flow equations.
\end{abstract}

\section{Introduction}

This work deals with the potential Kadomtsev-Petviashvili (pKP) hierarchy with dependent variable $\phi$ in an associative and noncommutative algebra, such that the product depends on additional variables 1 An important class is given by (Groenewold-) Moyal star products appearing in deformation quantization [1] and, more recently, in noncommutative field theories [2]. A new feature of corresponding Moyal deformed integrable equations 3 (see [12-15], for example) is the appearance of additional symmetries which are flows associated with the deformation parameters [16-19] and which extend the corresponding hierarchies. The extension of the Moyal-deformed KP hierarchy has been studied in [20-23]. Moreover, [23] also dealt with certain generalized star products (which involve iterated Moyal-type deformations, regarding the deformation parameters in each step as new variables on an equal footing with the original variables $\left.t_{1}, t_{2}, \ldots\right)$. The present work presents another perspective on these results, which in particular makes them easier accessible, and moreover extends them in several ways.

\footnotetext{
*(C)2007 by A. Dimakis and F. Müller-Hoissen

${ }^{1}$ The dependent KP variable is then $u=\partial_{t_{1}}(\phi)$, where $t_{1}$ is the first of the infinite set of independent variables $t_{1}, t_{2}, \ldots$ of the KP hierarchy.

${ }^{2}$ Here we mean Moyal deformations involving the independent variables which appear in the formulation of the respective differential equation or hierarchy. This has to be distinguished from the introduction of an additional 'momentum' partner for one of these variables (see [3-10], for example), or an additional pair of conjugate variables, as in [11].
} 
The 'deformation equations' that arise in this way are analogs of the equations which determine a socalled Seiberg-Witten map [2,24] in the context of 'noncommutative' gauge theory. These are maps from the classical to the (in the sense of deformation quantization [1]) 'quantized' theory. For the Moyal-deformed (matrix) $\mathrm{KdV}$ equation 3

$$
u_{t}=-u_{x x x}+3(u * u)_{x},
$$

such a 'deformation flow' is given by $[16,20]$

$$
u_{\theta}=-\frac{1}{2}\left[u, u_{x x}\right]_{*},
$$

where $\theta$ is the deformation parameter of the Moyal product $*$ in the space of smooth functions of $x$ and $t$, and $[u, v]_{*}:=u * v-v * u[$ To every solution of the classical $\mathrm{KdV}$ equation, the deformation equation determines a solution of the deformed KdV equation [16] (at least as a formal power series in the deformation parameter $\theta$ ). But there is more to it. The above deformation equation namely has the form of the (generalized) Heisenberg ferromagnet equation with 'time' $\theta$, and it indeed reduces to it 5 if we restrict $u$ to be independent of $t 6$ The Moyal deformation thus yields an unexpected link between different (classical) integrable equations 7

Another example is the Boussinesq equation

$$
\phi_{y y}=-\frac{1}{3} \phi_{x x x x}-2\left(\phi_{x} * \phi_{x}\right)_{x}+2\left[\phi_{x}, \phi_{y}\right],
$$

where $*$ is now the Moyal product with respect to the variables $x$ and $y$ and with deformation parameter $\theta^{\prime}$. In this case the corresponding deformation equation is the (deformed) potential $\mathrm{KdV}$ equation

$$
\phi_{\theta^{\prime}}=-\frac{1}{6} \phi_{x x x}-\phi_{x} * \phi_{x}
$$

(see also section 7.2 in [21]), where the deformation of the product disappears if $\phi_{y}=0$.

These examples demonstrate that the deformation flow equations are not at all exotic objects, in special cases they reproduce well-known integrable systems. This will be further supported in section 4 (second example) and in section 7 , where deformation parameters are identified as the usual evolution variables of the respective integrable systems.

There is another motivation for the exploration of deformations of the KP hierarchy. The famous Sato theory $[27,28]$ expresses the scalar KP hierarchy as Plücker relations of an infinite-dimensional Grassmannian. These are algebraic identities for Plücker coordinates. A crucial step towards this geometric

\footnotetext{
${ }^{3}$ Here $u_{t}$ denotes the partial derivative of $u$ with respect to $t$, and $u_{x x x}$ the third partial derivative with respect to $x$.

${ }^{4}$ Appendix A provides a FORM program $[25,26]$ to check the commutativity of the flows.

${ }^{5}$ Of course, we have to take $u$ as a matrix of functions which are multiplied using the Moyal product. In case of vanishing deformation, the noncommutativity of the matrix product then remains.

${ }^{6}$ The well-known relation with the familiar form $\vec{S}_{\tau}=\vec{S} \times \vec{S}_{x x}$ of the continuum Heisenberg ferromagnet equation (also known as Landau-Lifshitz equation) is obtained from $u_{\theta}=-\frac{1}{2}\left[u, u_{x x}\right]$ by choosing

$$
u=\left(\begin{array}{cc}
-S_{3} & S_{2}+\imath S_{1} \\
S_{2}-\imath S_{1} & S_{3}
\end{array}\right)
$$

with commuting functions satisfying $\vec{S}^{2}=1$, and $\theta=\imath \tau$.

${ }^{7}$ But this relation does not (at least not in a straight way) extend to a relation between the corresponding hierarchies, since the requirement that $u$ does not depend on certain $t_{n}$ in general leads to a too restrictive (and typically trivial) reduction of the (extended) KdV hierarchy. We should also mention that both, KdV and the Heisenberg ferromagnet equation, appear in branches of the AKNS system (see e.g. [20] and the references cited therein). Because of this reason the link between the two systems may not really come as a surprise. The Moyal link is, however, much more direct and of a very different nature. The AKNS framework suggests similar deformation relations between other integrable equations, but this will not be further elaborated in this work.
} 
interpretation is to express the dependent variable of the scalar KP hierarchy in terms of the so-called $\tau$ function. In the noncommutative case, where a direct analog of the $\tau$ function is not available, we obtained a result of similar nature [23,29]: the ('noncommutative') pKP equations are in correspondence with a class of identities in the algebra of quasi-symmetric functions. It turned out, however, that there are further similar classes of identities. In order to also translate these into differential equations, one is forced to introduce Moyal and the abovementioned generalized deformations. The corresponding deformations and extensions of the pKP hierarchy thus emerge in a natural way.

The Sato theory $[27,28]$ of the scalar KP hierarchy (and certain generalizations) achieves to linearize it on an infinite-dimensional space. In this spirit section 2 takes a quick step from a very general linear system to the noncommutative KP hierarchy. Here a matrix Riccati system plays a crucial role. This is further substantiated in section 3, which identifies the Riccati system as a special case of a universal system of ordinary differential equations in a 'weakly nonassociative' (WNA) algebra. This is based on the recent work in [29], see also [30,31]. After a first encounter in section 4 with a deformation equation of a Moyal deformed KP hierarchy, section 5 offers a more systematic treatment using the WNA framework. Section 6 then presents corresponding results for the more general deformations (generalized star products) mentioned above. Section 7 deals with a special case which puts the KP hierarchy in a new perspective: all evolution variables arise as deformation parameters! Finally, section 8 contains some conclusions.

\section{From a linear system to the KP hierarchy}

As already mentioned in the introduction, the Sato theory $[27,28]$ translates the KP hierarchy into a linear system of ordinary differential equations (which induce commuting flows on an infinite-dimensional Grassmannian). Let us go the inverse way and start with a linear system of ordinary differential equations 8

$$
Z_{t_{n}}=H^{n} Z \quad n=1,2, \ldots,
$$

where

$$
Z=\left(\begin{array}{c}
X \\
Y
\end{array}\right), \quad H=\left(\begin{array}{cc}
R & Q \\
S & L
\end{array}\right)
$$

Here $X, Y$ are $N \times N$, respectively $M \times N$ matrices with components from an associative algebra $\mathcal{R}$, the elements of which depend smoothly on independent variables $t_{n}, n \in \mathbb{N}$. Correspondingly, $L, Q, R, S$ are $M \times M, N \times M, N \times N$ and $M \times N$ matrices, respectively. Though we will concentrate on finitedimensional matrices $(M, N \in \mathbb{N}$ ), we may allow $M, N$ to be infinite (which is the case in the general Sato theory), provided that the product of such matrices is well-defined. The system (2.1) is compatible if $\left(H^{m}\right)_{t_{n}}=\left(H^{n}\right)_{t_{m}}$ for all $m, n$. This holds in particular if $H$ is constant, as assumed in the following. (2.1) is equivalent to the linear heat hierarchy

$$
Z_{t_{n}}=\left(\partial_{t_{1}}\right)^{n} Z \quad n=2,3, \ldots,
$$

supplemented with

$$
Z_{t_{1}}=H Z
$$

This is solved by

$$
Z=e^{\xi(H)} Z_{0}, \quad \xi(H):=\sum_{n \geq 1} t_{n} H^{n},
$$

\footnotetext{
${ }^{8}$ If $H$ is invertible, it may be possible to extend the system to $n \in \mathbb{Z}$, thus adding a 'negative hierarchy'. But see also the remark in section 3.5.4 of [28] concerning possible obstructions in the infinite-dimensional case due to nonvanishing Kac-Peterson cocycles.
} 
where $Z_{0}$ is constant, i.e. independent of $t_{1}, t_{2}, \ldots$ Setting

$$
\left(\begin{array}{cc}
R_{n} & Q_{n} \\
S_{n} & L_{n}
\end{array}\right):=H^{n}
$$

we obtain the recursion relations

$$
\begin{aligned}
& L_{m+n}=L_{m} L_{n}+S_{m} Q_{n}, \quad Q_{m+n}=R_{m} Q_{n}+Q_{m} L_{n}, \\
& R_{m+n}=R_{m} R_{n}+Q_{m} S_{n}, \quad S_{m+n}=L_{m} S_{n}+S_{m} R_{n},
\end{aligned}
$$

where $L_{1}=L, Q_{1}=Q, R_{1}=R, S_{1}=S$. The linear system (2.1) decomposes into

$$
X_{t_{n}}=R_{n} X+Q_{n} Y, \quad Y_{t_{n}}=S_{n} X+L_{n} Y .
$$

Let $*$ be any (other) associative product in $\mathcal{R}$ which, extended to a matrix product, satisfies the relations

$$
(L A) * B=L(A * B), \quad\left(A^{\prime} L\right) * B^{\prime}=A^{\prime} *\left(L B^{\prime}\right), \quad A^{\prime \prime} *\left(B^{\prime \prime} L\right)=\left(A^{\prime \prime} * B^{\prime \prime}\right) L,
$$

and correspondingly with $L$ replaced by $Q, R$ and $S$. Here $A, A^{\prime}, A^{\prime \prime}, B, B^{\prime}, B^{\prime \prime}$ are matrices with entries in $\mathcal{R}$ and appropriate dimensions, so that the matrix multiplications in these equations are well-defined and in particular fit to the dimensions of $L, Q, R, S$, respectively. By induction, using the recursion relations (2.7), one obtains the corresponding relations for $L_{n}, Q_{n}, R_{n}, S_{n}, n=2,3, \ldots$ We shall also assume that the partial derivatives with respect to the variables $t_{n}$ are derivations of the product $*$.

Proposition 1 For all $\mathbf{t}=\left(t_{1}, t_{2}, \ldots\right)$ for which $X$ possesses an inverse $X^{*-1}$ (with respect to $*$ ),

$$
\phi:=Y * X^{*-1}
$$

solves the $p K P$ hierarchy in the algebra $\mathcal{A}$ of $M \times N$ matrices with entries in $\mathcal{R}$ and product

$$
A \cdot B:=A * Q B
$$

Proof: This can be proved using a functional representation of the pKP hierarchy, see [32]. An alternative proof will be given in this work, based on the next proposition and results recalled in section 3 .

Proposition 2 The linear system (2.1) implies the matrix Riccati system

$$
\phi_{t_{n}}=S_{n}+L_{n} \phi-\phi R_{n}-\phi * Q_{n} \phi \quad n=1,2, \ldots .
$$

With suitable restrictions imposed on $(\mathcal{R}, *)$ (as specified in the proof), the two systems are in fact equivalent.

Proof: Using the definition (2.10) and the derivation property of the partial derivatives $\partial_{t_{n}}$, we have

$$
Y_{t_{n}}=(\phi * X)_{t_{n}}=\phi_{t_{n}} * X+\phi * X_{t_{n}}
$$

If (2.1) holds, and thus (2.8), a direct calculation leads to (2.12). Conversely, if (2.12) holds, the above equation becomes

$$
Y_{t_{n}}=S_{n} X+L_{n} Y+\phi *\left(X_{t_{n}}-R_{n} X-Q_{n} Y\right),
$$

and thus $Y_{t_{n}}-S_{n} X-L_{n} Y=Y * C_{n}$, where $C_{n}:=X^{*-1} *\left(X_{t_{n}}-R_{n} X-Q_{n} Y\right)$. Hence

$$
Z_{t_{n}}=H^{n} Z+Z * C_{n} .
$$


The integrability conditions $Z_{t_{m} t_{n}}=Z_{t_{n} t_{m}}$ now demand that $C_{m, t_{n}}-C_{n, t_{m}}+\left[C_{n}, C_{m}\right]_{*}=0$, which means that the curvature of the connection $C=\sum_{n>1} C_{n} d t_{n}$ vanishes. If this implies the existence of a gauge transformation $Z^{\prime}=Z * G$ which transforms the connection to zero (as in the familiar case where $(\mathcal{R}, *)$ is the algebra of functions on a chart of some manifold), we obtain $Z_{t_{n}}^{\prime}=H^{n} Z^{\prime}$, which is the linear system (2.1).

Elimination 9 of $L, R, S$ from the equations (2.12) (using also equations derived from them by differentiations) leads to the pKP hierarchy for $\phi$ in $(\mathcal{A}, \cdot)$. A corresponding proof is given in section 3

Example. In the special case where $S=0$, we have

$$
R_{n}=R^{n}, \quad L_{n}=L^{n},
$$

and $Q_{n+1}=Q L^{n}+R Q_{n}$. By induction, this leads to

$$
Q_{n}=\sum_{k=0}^{n-1} R^{k} Q L^{n-k-1} .
$$

In order that $H$ (with $S=0$ ) can be block-diagonalized by a matrix

$$
T=\left(\begin{array}{cc}
I_{N} & -K \\
0 & I_{M}
\end{array}\right)
$$

i.e. $T^{-1} H T$ is block-diagonal, we need the condition

$$
Q=R K-K L .
$$

Then (2.14) becomes a telescoping sum which results in

$$
Q_{n}=R^{n} K-K L^{n} .
$$

In this case the matrix Riccati system (2.12) is solved by

$$
\phi=e^{\xi(L)} \phi_{0} *\left(e^{\xi(R)}\left(I_{N}+K \phi_{0}\right)-K e^{\xi(L)} \phi_{0}\right)^{*-1},
$$

where $\phi_{0}=Y_{0} * X_{0}^{*-1}$. Note that this solution of the pKP hierarchy in $(\mathcal{A}, \cdot)$ is 'universal' in the sense that we did not have to specify the associative product $*$.

(2.12) with (2.14) implies the so-called Sato system [28,33]

$$
\mathcal{W}(i, j)_{t_{n}}=\mathcal{W}(i+n, j)-\mathcal{W}(i, j+n)-\sum_{k=0}^{n-1} \mathcal{W}(i, k) * Q \mathcal{W}(n-k-1, j),
$$

where 10

$$
\mathcal{W}(i, j):=L^{i} \phi R^{j} \quad i, j=0,1,2, \ldots
$$

(see also [31]). If (2.16) holds, the system (2.19) simplifies to

$$
\mathcal{W}(i, j)_{t_{n}}=\mathcal{W}(i+n, j)-\mathcal{W}(i, j+n)-\mathcal{W}(i, n) * K \mathcal{W}(0, j)+\mathcal{W}(i, 0) * K \mathcal{W}(n, j)
$$

\footnotetext{
${ }^{9}$ The 'elimination' does not make use of any special, e.g. matrix, properties of $L, R, S$, but rather treats them as abstract algebraic symbols. This includes a lot of freedom so that the Riccati system (2.12) reaches large classes of solutions of the pKP hierarchy, if not even all. This remark applies correspondingly to the 'elimination procedure' in section 4

${ }^{10}$ If $L$ or $R$ is a finite-dimensional matrix, there are of course algebraic relations among the $\mathcal{W}(i, j)$ (e.g., as a consequence of the Cayley-Hamilton theorem).
} 
The Sato system is well-known to be linearizable, see also the following remark.

Remark. If $S \neq 0$, (2.20) leads to a generalization of the Sato system, which can again be linearized by writing $\mathcal{W}=\mathcal{Y} * \mathcal{X}^{*-1}$. This leads to the linear system

$$
\mathcal{Z}_{t_{n}}=\mathcal{H}^{n} \mathcal{Z} \quad \text { where } \quad \mathcal{Z}=\left(\begin{array}{c}
\mathcal{X} \\
\mathcal{Y}
\end{array}\right), \quad \mathcal{H}=\left(\begin{array}{cc}
\Lambda^{T} & \mathbf{e}_{0} \mathbf{e}_{0}^{T} \otimes Q \\
\mathcal{S} & \Lambda
\end{array}\right)
$$

with $\mathcal{S}(i, j)=L^{i} S R^{j}, \mathbf{e}_{0}^{T}=(1,0, \ldots)$, and the shift operator matrix

$$
\Lambda=\left(\begin{array}{ccccc}
0 & 1 & 0 & & \cdots \\
0 & 0 & 1 & 0 & \cdots \\
\vdots & \ddots & \ddots & \ddots & \ddots
\end{array}\right)
$$

If $S=0$, this provides a linearization of the Sato system.

Remark. Let $Q=V U^{T}$ with a constant $M \times m$ matrix $U$ and a constant $N \times m$ matrix $V$, and such that $A * Q B=A V * U^{T} B$. If $\phi$ solves the pKP hierarchy in $(\mathcal{A}, \cdot)$ (with the product (2.11) ), then

$$
\varphi:=U^{T} \phi V
$$

solves the pKP hierarchy in the algebra of $m \times m$ matrices with product $*$.

Let $\mathcal{R}$ be the algebra of smooth complex functions of $t_{1}, t_{2}, \ldots$, and $*$ the original product in $\mathcal{R}$. Then, for $m=1$,

$$
\varphi=\operatorname{tr}(Q \phi)
$$

solves the scalar pKP hierarchy. Using

$$
Q \phi=X_{t_{1}} X^{-1}-R
$$

which results from (2.8) (and reminds us of a matrix Cole-Hopf transformation, see also [30, 32, 34]), we obtain

$$
\varphi=\operatorname{tr}\left(X_{t_{1}} X^{-1}\right)-\operatorname{tr}(R)=(\log \tau)_{t_{1}}-\operatorname{tr}(R)
$$

with

$$
\tau=\operatorname{det}(X) .
$$

In particular, any solution of the matrix Riccati system (2.12) with $\operatorname{rank}(Q)=1$ determines in this way a $\tau$ function (and then a solution) of the scalar KP hierarchy. The solution (2.18) of the matrix pKP hierarchy leads in this way to a large set of solutions of the scalar KP hierarchy (see also [30,32,35] and the references therein).

\section{A nonassociative algebraic structure underlying the Riccati system}

Let $(\mathcal{A}, \cdot)$ denote the algebra of $M \times N$ matrices considered in the preceding section. In this algebra we introduce the sequence of products

$$
A \circ_{n} B=A * Q_{n} B \quad n=1,2, \ldots
$$


(so that $A \circ_{1} B=A \cdot B$ ), which are combined associative, i.e.,

$$
A \circ_{n}\left(B \circ_{m} C\right)=\left(A \circ_{n} B\right) \circ_{m} C .
$$

We augment the algebra $\mathcal{A}$ with an element $\nu$ such that

$$
\nu \circ_{n} \nu=-S_{n}, \quad \nu \circ_{n} A=L_{n} A, \quad A \circ_{n} \nu=-A R_{n} .
$$

Let $\mathbb{A}$ denote the resulting algebra. From the recursion relations $(2.7)$ we obtain

$$
a \circ_{m+n} b=a \circ_{m}\left(\nu \circ_{n} b\right)-\left(a \circ_{m} \nu\right) \circ_{n} b
$$

for all $a, b \in \mathbb{A}$. This relation determines all the products $\circ_{n}$ in terms of $\circ_{1}$. If $a \circ_{2} b \neq 0$ for some $a, b \in \mathbb{A}$, then $\mathbb{A}$ is not associative. Nonassociativity only enters through the augmented element $\nu$. This motivates the following definition [29] (see also [30]).

An algebra $(\mathbb{A}, \circ)$ (over a commutative ring) is called weakly nonassociative (WNA) if it is not associative, but the associator $(a, b \circ c, d)$ vanishes for all $a, b, c, d \in \mathbb{A}$ (where the associator is defined as $(a, b, c)=(a \circ b) \circ c-a \circ(b \circ c))$. The middle nucleus $\mathbb{A}^{\prime}=\{b \in \mathbb{A} \mid(a, b, c)=0 \forall a, c \in \mathbb{A}\}$, which is an associative subalgebra, is then also an ideal in $\mathbb{A}$. With respect to an element $f \in \mathbb{A}, f \notin \mathbb{A}^{\prime}$, we define the products $a \circ_{1} b=a \circ b$ and

$$
a \circ_{n+1} b=a \circ\left(f \circ_{n} b\right)-(a \circ f) \circ_{n} b \quad n=1,2, \ldots .
$$

As a consequence of the WNA condition, these products only depend on the equivalence class $[f]$ of $f$ in the quotient space $\mathbb{A} / \mathbb{A}^{\prime}$. Next we recall a central result from [29].

Theorem 1 Let $(\mathbb{A}, \circ)$ be any WNA algebra, the elements of which depend smoothly on independent variables $t_{1}, t_{2}, \ldots$. Then the flows of the system of ordinary differential equations

$$
f_{t_{n}}=f \circ_{n} f \quad n=1,2, \ldots
$$

in $\mathbb{A}$ commute and imply that $-f_{t_{1}} \in \mathbb{A}^{\prime}$ solves the KP hierarchy in the associative subalgebra $\left(\mathbb{A}^{\prime}, \circ\right)$.

The algebra $\mathbb{A}$ introduced above is easily seen to be WNA. Setting

$$
f=\nu-\phi,
$$

so that $[f]=[\nu]$, then (3.5) coincides with (3.4) for $m=1$. Furthermore, (3.5) implies (3.4) (see proposition 3.3 in [29]). Assuming that $\nu$ is constant (i.e., independent of $t_{1}, t_{2}, \ldots$ ), (2.12) becomes (3.6). By application of the theorem, it follows that if $\phi$ solves the Riccati system (2.12), then it also solves the pKP hierarchy in $(\mathcal{A}, \cdot)$.

Of course, there are other realizations of WNA algebras than given by the class of examples which we encountered in this section, in particular a realization in terms of quasi-symmetric functions [29] and a realization in terms of formal pseudodifferential operators [30] (which allows to make contact with the Gelfand-Dickey formalism [36] of the KP hierarchy). 


\section{The case of a Moyal star product}

New structures appear if the product $*$ depends on additional variables. Of particular interest is the case of a (Groenewold-) Moyal star product

$$
g_{1} * g_{2}:=\mathbf{m} \exp \left(\frac{1}{2} \sum_{m, n \geq 1} \theta_{m n} \partial_{t_{m}} \otimes \partial_{t_{n}}\right) g_{1} \otimes g_{2}
$$

with antisymmetric (deformation) parameters $\theta_{m n}$ and $\mathbf{m}\left(g_{1} \otimes g_{2}\right)=g_{1} g_{2} 11$ The product extends to matrices by combining the ordinary matrix product with the Moyal product of the components. So far this just gives a class of examples of noncommutative associative products to which our results in the previous sections apply. But now we can think of the deformation parameters as new variables which describe deformation flows of the deformed KP hierarchy. Since

$$
\left(g_{1} * g_{2}\right)_{\theta_{m n}}=g_{1, \theta_{m n}} * g_{2}+g_{1} * g_{2, \theta_{m n}}+\frac{1}{2}\left(g_{1, t_{m}} * g_{2, t_{n}}-g_{1, t_{n}} * g_{2, t_{m}}\right),
$$

there is a non-trivial coupling of these flows to those of the pKP hierarchy 12 By considering deformation flows we are actually leaving the framework of the KP hierarchy in a fixed algebra (i.e., with a fixed product). Instead we are dealing with a family of KP hierarchies, parametrized by the deformation parameters entering the product. This point of view is supported by the fact that the KP hierarchy possesses solutions which can be expressed without the need of specifying the concrete form of the product, see the example in section 2 In the following we will not be so careful to distinguish between a deformed KP hierarchy and the family of deformed KP hierarchies. It is the latter which we are studying.

Let us try to extend the linear system (2.1) by supplementing it with compatible linear ordinary differential equations in the variables $\theta_{m n}$. Using only what we already have at our disposal, we should build the corresponding vector field in terms of $H$. But because of the antisymmetry of the variables $\theta_{m n}$, we are left with the apparently trivial choice

$$
Z_{\theta_{m n}}=0 \quad m, n=1,2, \ldots .
$$

Nevertheless, this leads to non-trivial results since now (2.10) involves the deformation parameters through the product. In fact, we obtain

$$
0=Y_{\theta_{m n}}=(\phi * X)_{\theta_{m n}}=\phi_{\theta_{m n}} * X+\frac{1}{2}\left(\phi_{t_{m}} * X_{t_{n}}-\phi_{t_{n}} * X_{t_{m}}\right),
$$

and thus

$$
\phi_{\theta_{m n}}=\frac{1}{2}\left(\phi_{t_{n}} * X_{t_{m}}-\phi_{t_{m}} * X_{t_{n}}\right) * X^{*-1} .
$$

By use of (2.8), this implies the deformation flow equations

$$
\phi_{\theta_{m n}}=\frac{1}{2}\left(\phi_{t_{n}} *\left(R_{m}+Q_{m} \phi\right)-\phi_{t_{m}} *\left(R_{n}+Q_{n} \phi\right)\right) .
$$

\footnotetext{
${ }^{11}$ The tensor product has to be taken over $\mathbb{K}\left[\left[\left\{\theta_{m n}\right\}\right]\right]$ where $\mathbb{K}$ is $\mathbb{R}$ or $\mathbb{C}$.

${ }^{12}$ If $\tilde{\mathbf{m}}$ denotes any product which depends on a parameter $\theta$, then $\partial_{\theta}\left(\tilde{\mathbf{m}}\left(g_{1}, g_{2}\right)\right)=\tilde{\mathbf{m}}\left(\partial_{\theta}\left(g_{1}\right), g_{2}\right)+\tilde{\mathbf{m}}\left(g_{1}, \partial_{\theta}\left(g_{2}\right)\right)+$ $\partial_{\theta}(\tilde{\mathbf{m}})\left(g_{1}, g_{2}\right)$. In case of the Moyal product, we have $\partial_{\theta_{m n}}(\tilde{\mathbf{m}})\left(g_{1}, g_{2}\right)=\frac{1}{2}\left(\tilde{\mathbf{m}}\left(g_{1, t_{m}}, g_{2, t_{n}}\right)-\tilde{\mathbf{m}}\left(g_{1, t_{n}}, g_{2, t_{m}}\right)\right)$, so that $\partial_{\theta_{m n}}(\tilde{\mathbf{m}})$ can be expressed in terms of $\tilde{\mathbf{m}}$. This property is crucial for the following calculations.
} 
With the help of the Riccati system (2.12), these are converted into the ordinary differential equations

$$
\begin{aligned}
\phi_{\theta_{m n}}= & \frac{1}{2}\left(S_{n} R_{m}-S_{m} R_{n}+\left(S_{n} Q_{m}-S_{m} Q_{n}\right) \phi+L_{n} \phi R_{m}-L_{m} \phi R_{n}-\phi\left[R_{n}, R_{m}\right]\right. \\
& +L_{n} \phi * Q_{m} \phi-L_{m} \phi * Q_{n} \phi-\phi *\left(R_{n} Q_{m}-R_{m} Q_{n}\right) \phi-\phi * Q_{n} \phi R_{m}+\phi * Q_{m} \phi R_{n} \\
& \left.-\phi * Q_{n} \phi * Q_{m} \phi+\phi * Q_{m} \phi * Q_{n} \phi\right) \quad m, n=1,2, \ldots .
\end{aligned}
$$

Because of the commutativity of flows on the level of the linear system, we should expect these deformation flows to commute with each other, and also with the flows of the Riccati system (2.12). There is a subtlety, however, which arises from the elimination of the terms $X_{t_{n}} * X^{*-1}$ in the step from (4.5) to (4.6). Whereas any of the flows (4.5) indeed commutes with any of the flows of the Riccati system (2.12) without further conditions, the commutativity of (4.6) or (4.7) for fixed $m, n$ with the $t_{r}$-flow of (2.12) also requires the two equations of (2.12) with evolution variables $t_{m}$ and $t_{n}$. We will refer to this feature as 'conditional commutativity'.

Eliminating $L, R, S$ (regarded as abstract algebraic symbols) from the above equations, results in deformation equations for $\phi$, the simplest of which is

$$
\phi_{\theta_{12}}=\frac{1}{6}\left(\phi_{t_{3}}-\phi_{t_{1} t_{1} t_{1}}\right)-\phi_{t_{1}} * Q \phi_{t_{1}} .
$$

This already requires a lengthy calculation (see also appendix B), which asks for a method to achieve such results in a more systematic and efficient way, a problem addressed in the following section. The deformed pKP hierarchy together with its deformation equations will be called 'extended (deformed) pKP hierarchy'. The deformation flows commute with each other and with those of the pKP hierarchy conditionally in the sense explained above.

An equation like (4.8) is not a symmetry of the pKP hierarchy in a given algebra (which would mean fixing the deformation parameters in the product (4.1)), but rather a symmetry of the family of pKP hierarchies, which is parametrized in terms of the deformation parameters. The corresponding flow maps solutions of one pKP hierarchy to solutions of another pKP hierarchy (with different values of the parameters $\theta_{m n}$ ).

Remark. If $S=0$ and $Q=R K-K L$, we have the solution (2.18) of the Moyal-deformed matrix Riccati system and thus the Moyal-deformed matrix pKP hierarchy (with matrix product modified by $Q$ ). It also solves all the deformation equations, like (4.8).

Remark. If $\phi$ solves the extended deformed pKP hierarchy in the matrix algebra with product $A \cdot B=$ $A * Q B$, then (2.24) solves the extended deformed pKP hierarchy in the algebra of $m \times m$ matrices with entries in the Moyal algebra. (4.8) then leads to

$$
\varphi_{\theta_{12}}=\frac{1}{6}\left(\varphi_{t_{3}}-\varphi_{t_{1} t_{1} t_{1}}\right)-\varphi_{t_{1}} * \varphi_{t_{1}} .
$$

This equation (for $m=1$ ) first appeared in [20], see also [21-23].

Example. We show that, in a special case, a deformation flow equation reproduces the potential KdV equation such that the deformation parameter takes the role of its (usual) time variable. Let us consider only the first two equations of the linear system, i.e.

$$
Z_{x}=H Z, \quad Z_{y}=H^{2} Z,
$$

where $x=t_{1}$ and $y=t_{2}$. Assuming moreover

$$
\phi_{y}=0,
$$


this leads to

$$
\phi_{x}=S+L \phi-\phi R-\phi Q \phi, \quad S_{2}+L_{2} \phi-\phi R_{2}-\phi Q_{2} \phi=0,
$$

which is not affected by the deformation so that only the original product appears. From $Z_{\theta}=0$, where $\theta=\theta_{12}$, we find

$$
0=Y_{\theta}=\phi_{\theta} * X+\frac{1}{2} \phi_{x} * X_{y}
$$

and thus, by use of (4.10) (decomposed as in (2.8)),

$$
\phi_{\theta}=-\frac{1}{2} \phi_{x}\left(R_{2}+Q_{2} \phi\right) .
$$

A straightforward but tedious calculation, eliminating $L, R, S$ from our three equations for $\phi$, leads to 13

$$
\phi_{\theta}=-\frac{1}{8} \phi_{x x x}-\frac{3}{4} \phi_{x} Q \phi_{x} .
$$

This is the potential KdV equation, where the deformation parameter $\theta$ plays the role of the evolution 'time' variable $t 14$ Here the KdV flow originates from a Moyal deformation! See also section 7.1 in [21] for related results. From (4.10) and $\phi_{y}=0$, we obtain $Y_{y}=\phi * X_{y}=Y * C$ with an $N \times N$ matrix $C$ such that $R_{2} X+Q_{2} Y=X * C$. Furthermore, we have $S_{2} X+L_{2} Y=Y * C$, which combines with the last equation to

$$
H^{2} Z=Z * C
$$

where on the right hand side both parts $(X$ and $Y$ ) of $Z$ are multiplied by $C$. Since the solution of the above linear system is given by

$$
Z=e^{x H+y H^{2}} Z_{0}
$$

it follows that

$$
H^{2} Z_{0}=Z_{0} C
$$

which is a condition on the initial data given by $Z_{0}$. Conversely, this condition implies $\phi_{y}=0$ by use of (4.10). In conclusion, any solution of the linear system (4.10) and $Z_{\theta}=0$, with initial data satisfying $H^{2} Z_{0}=Z_{0} C$ with some $N \times N$ matrix $C$, solves the potential $\mathrm{KdV}$ equation (4.15).

\section{The WNA version of the Moyal deformation equations}

Using (3.7) and the WNA rules of section 3, where now $*$ is chosen to be the Moyal product defined in the preceding section, the system (4.6) can be expressed in the form

$$
f_{\theta_{m n}}=\frac{1}{2}\left(f_{t_{m}} \circ_{n} f-f_{t_{n}} \circ_{m} f\right)=\frac{1}{2}\left(\left(f \circ_{m} f\right) \circ_{n} f-\left(f \circ_{n} f\right) \circ_{m} f\right) \quad m, n=1,2, \ldots,
$$

\footnotetext{
${ }^{13}$ In order to check that this equation holds, one first eliminates the derivatives of $\phi$ by use of our previous equations for $\phi_{x}$ and $\phi_{\theta}$. The resulting algebraic equation is then satisfied as a consequence of the algebraic Riccati equation in 4.12) which resulted from setting $\phi_{y}=0$. This is easily verified using FORM.

${ }^{14}$ After suitable rescalings of the variables, it takes the form 1.1 .
} 
by use of (3.6). With the help of (3.4) this can be rewritten as

$$
f_{\theta_{m n}}=\frac{1}{2}\left(f \circ_{m}\left(f \circ \circ_{n} f\right)-f \circ_{n}\left(f \circ \circ_{m} f\right)\right) .
$$

Next we address the commutativity of these flows, and also with those given by (3.6), by solely using general properties of WNA algebras. With this step we proceed beyond the special realization of a WNA algebra given in section 3 .

Lemma 1 Let $\mathbb{A}$ be a Moyal-deformed WNA algebra and $f \in \mathbb{A}$ such that $\partial_{t_{n}}(f) \in \mathbb{A}^{\prime}$ and $\partial_{\theta_{m n}}(f) \in \mathbb{A}^{\prime}$ for all $m, n=1,2, \ldots$. Then, for $k=1,2, \ldots$, we have

$$
\partial_{\theta_{m n}}\left(a \circ_{k} b\right)=\partial_{\theta_{m n}}(a) \circ_{k} b+a \circ_{k} \partial_{\theta_{m n}}(b)+\frac{1}{2}\left(\partial_{t_{m}}(a) \circ_{k} \partial_{t_{n}}(b)-\partial_{t_{n}}(a) \circ_{k} \partial_{t_{m}}(b)\right) .
$$

Proof: by induction on $k$, using (3.5).

Proposition 3 For any Moyal-deformed WNA algebra, the flows (5.2) commute with the flows (3.6) and with each othen 15

Proof: As a consequence of (3.6) and (5.2), the assumptions of the preceding lemma are fulfilled. Hence

$$
\left(f_{t_{r}}\right)_{\theta_{m n}}=\left(f \circ \circ_{r} f\right)_{\theta_{m n}}=f_{\theta_{m n}} \circ_{r} f+f \circ_{r} f_{\theta_{m n}}+\frac{1}{2}\left(f_{t_{m}} \circ_{r} f_{t_{n}}-f_{t_{n}} \circ_{r} f_{t_{m}}\right),
$$

which has to be further worked out with the help of (5.2), and (3.6) for $m$ and $n$. A similar calculation evaluates $\left(f_{\theta_{m n}}\right)_{t_{r}}$. Taking the difference of the results leads to

$$
\left(f_{t_{r}}\right)_{\theta_{m n}}-\left(f_{\theta_{m n}}\right)_{t_{r}}=\frac{1}{2}\left(F_{m n r}-F_{n m r}\right)
$$

with

$$
\begin{aligned}
F_{m n r}:= & f \circ_{m}\left(\left(f \circ_{n} f\right) \circ_{r} f\right)-f \circ_{m}\left(f \circ_{n}\left(f \circ_{r} f\right)\right)+f \circ_{r}\left(f \circ_{m}\left(f \circ_{n} f\right)\right) \\
& -\left(f \circ_{r} f\right) \circ_{m}\left(f \circ_{n} f\right)+\left(f \circ_{m} f\right) \circ_{r}\left(f \circ_{n} f\right)-f \circ_{m}\left(\left(f \circ_{r} f\right) \circ_{n} f\right),
\end{aligned}
$$

which turns out to vanish by application of proposition 3.3 in [29] (which is (3.4) with $\nu$ replaced by $f$ ). The commutativity of two deformation flows, under the condition that the associated equations of the hierarchy (3.6) are satisfied, is verified in the same way with little more efforts. We omit the details since this result can also be deduced from the more general proposition 7 below (since $f_{\theta_{m n}}=\left(f_{t_{m n}}-f_{t_{n m}}\right) / 2$ ).

In the preceding section we were led to the problem of eliminating $L, R, S$ from the deformation flow equations (4.7), in order to obtain partial differential equations involving $\phi$ (and $Q$ ) only. Since according to section 3 they are encoded in the action of the constant augmented element $\nu=f+\phi$, this means we have to look for equations resulting from (5.2) which do not contain a 'bare' $f$, i.e. an $f$ without a partial derivative acting on it (note that $f_{t_{n}}=-\phi_{t_{n}}$ ). 16 Unfortunately there is no simple way to extract such equations from (5.2). The next result is a generalization of Lemma 5.1 in [29]. Recall that $\circ$ and $\circ_{1}$ denote the same product.

\footnotetext{
${ }^{15}$ More precisely, this is a 'conditional commutativity' as discussed in section 4 The commutativity of the two flows with evolution variables $t_{m n}$ and $t_{r s}$ requires also the four equations of (3.6) with evolution variables $t_{m}, t_{n}, t_{r}, t_{s}$.

${ }^{16}$ In particular, these restrictions rule out expressions involving 'higher' products $\circ_{n}, n>1$, of elements of $\mathbb{A}^{\prime}$, since they contain bare $f$ 's, see the definition 3.5 .
} 
Lemma 2 In a WNA algebra with the products $\circ_{n}$ defined in (3.5), the following identities hold,

$$
\begin{aligned}
& f \circ_{n} a=L_{f}^{n} a-\sum_{k=1}^{n-1} p_{n-k} \circ L_{f}^{k-1} a \\
& a \circ_{n} f=(-1)^{n+1} R_{f}^{n} a-\sum_{k=1}^{n-1}(-1)^{k}\left(R_{f}^{k-1} a\right) \circ p_{n-k} .
\end{aligned}
$$

where

$$
p_{n}:=f \circ_{n} f,
$$

and $L_{f}, R_{f}$ denote, respectively, left and right action by $f$.

Proof: By use of proposition 3.3 in [29] (which is (3.4) with $\nu$ replaced by $f$ ) we have

$$
\sum_{k=1}^{n-1} p_{n-k} \circ L_{f}^{k-1} a=\sum_{k=1}^{n-1}\left(f \circ_{n-k} f\right) \circ_{1} L_{f}^{k-1} a=\sum_{k=1}^{n-1}\left(f \circ_{n-k}\left(f \circ_{1} L_{f}^{k-1} a\right)-f \circ_{n-k+1} L_{f}^{k-1} a\right),
$$

which is a telescoping sum that collapses to $L_{f}^{n} a-f \circ_{n} a$. This proves the first assertion, and the second is verified in the same way.

As an application of this lemma, we find

$$
f \circ_{m}\left(f \circ_{n} f\right)=h_{m}^{(n)}-\sum_{k=1}^{m-1} p_{m-k} \circ h_{k-1}^{(n)},
$$

where we introduced

$$
h_{m}^{(n)}=L_{f}^{m} p_{n} \quad m=0,1, \ldots, \quad n=1,2, \ldots
$$

Note that

$$
h_{m}^{(1)}=L_{f}^{m+1} f=: h_{m+1}, \quad h_{0}^{(n)}=p_{n} .
$$

With the help of (5.7) we can now rewrite (5.2). Next we look for efficient formulae to compute the expressions (5.8).

Proposition 4 As a consequence of (3.6) we have 17

$$
h_{m}^{(r+n)}=h_{r+m}^{(n)}+h_{m+n}^{(r)}-\partial_{t_{r}} h_{m}^{(n)}+\sum_{k=1}^{m} h_{k-1}^{(r)} \circ h_{m-k}^{(n)}-\sum_{k=1}^{r-1} h_{m}^{(r-k)} \circ h_{k-1}^{(n)}-\sum_{k=1}^{n-1} h_{m}^{(n-k)} \circ h_{k-1}^{(r)}
$$

Proof: First we note that

$$
\partial_{t_{r}} h_{1}^{(n)}=f_{t_{r}} \circ\left(f \circ_{n} f\right)+f \circ\left(f_{t_{r}} \circ_{n} f+f \circ_{n} f_{t_{r}}\right)=p_{r} \circ p_{n}+f \circ\left(p_{r} \circ_{n} f+f \circ_{n} p_{r}\right) .
$$

With the help of $f \circ_{r+n} f=f \circ_{r} p_{n}-p_{r} \circ_{n} f$ (proposition 3.3 in [29]), this is recognized as the $m=1$ case of

$$
\partial_{t_{r}} h_{m}^{(n)}=\sum_{k=1}^{m} h_{k-1}^{(r)} \circ h_{m-k}^{(n)}+L_{f}^{m}\left(f \circ_{r}\left(f \circ_{n} f\right)+f \circ_{n}\left(f \circ_{r} f\right)\right)-h_{m}^{(r+n)},
$$

\footnotetext{
${ }^{17}$ For $r=1$ this becomes $h_{m}^{(n+1)}=h_{m+1}^{(n)}+h_{m+n+1}-\partial_{t_{1}} \circ h_{m}^{(n)}+\sum_{k=1}^{m} h_{k} \circ h_{m-k}^{(n)}-\sum_{k=1}^{n-1} h_{m}^{(n-k)} \circ h_{k}$, a relation which already appeared, with different notation, as (5.16) in [21] and as (4.14) in [23].
} 
which is then easily proved by induction on $m$ using $\partial_{t_{r}} h_{m+1}^{(n)}=\partial_{t_{r}}\left(f \circ h_{m}^{(n)}\right)=p_{r} \circ h_{m}^{(n)}+f \circ \partial_{t_{r}} h_{m}^{(n)}$. Finally we make use of (5.7) to translate the last formula into (5.10).

For $n=1,(5.10)$ reduces to

$$
h_{m}^{(r+1)}=h_{m+r+1}+h_{m+1}^{(r)}-\partial_{t_{r}} h_{m+1}+\sum_{k=1}^{m} h_{k-1}^{(r)} \circ h_{m-k+1}-\sum_{k=1}^{r-1} h_{m}^{(r-k)} \circ h_{k},
$$

which allows a recursive computation of the $h_{m}^{(n)}$ in terms of the $h_{k}$ and their derivatives. In particular, from (5.7) and 5.11) we obtain

$$
\begin{aligned}
& f \circ_{1}\left(f \circ_{2} f\right)=h_{1}^{(2)}=2 h_{3}-\left(h_{2}\right)_{t_{1}}+p_{1} \circ p_{1}, \\
& f \circ_{2}\left(f \circ_{1} f\right)=h_{2}^{(1)}-p_{1} \circ h_{0}^{(1)}=h_{3}-p_{1} \circ p_{1},
\end{aligned}
$$

so that

$$
f \circ_{1}\left(f \circ_{2} f\right)-f \circ_{2}\left(f \circ_{1} f\right)=h_{3}-\left(h_{2}\right)_{t_{1}}+2 p_{1} \circ p_{1} .
$$

Theorem 5.3 in [29], which makes use of the hierarchy equations (3.6), expresses $h_{n}$ as

$$
h_{n}=\mathbf{p}_{n}(\tilde{\partial}) f
$$

in terms of the elementary Schur polynomial $\mathbf{p}_{n}$ and $\tilde{\partial}:=\left(\partial_{t_{1}}, \partial_{t_{2}} / 2, \partial_{t_{3}} / 3, \ldots\right)$. In particular, we find

$$
h_{2}=\frac{1}{2} f_{t_{2}}+\frac{1}{2} f_{t_{1} t_{1}}, \quad h_{3}=\frac{1}{3} f_{t_{3}}+\frac{1}{2} f_{t_{2} t_{1}}+\frac{1}{6} f_{t_{1} t_{1} t_{1}} .
$$

Thus we obtain

$$
f_{\theta_{12}}=\frac{1}{6}\left(f_{t_{3}}-f_{t_{1} t_{1} t_{1}}\right)+f_{t_{1}} \circ f_{t_{1}},
$$

which, by use of (3.7), reproduces our previous equation (4.8). The advantage is that now we have a systematic way to derive such deformation equations. This method is considerably simpler than that described in [23].

\section{Beyond Moyal deformation}

Instead of the Moyal deformation, let us consider the deformation 18

$$
g_{1} *_{2} g_{2}:=\mathbf{m}_{2}\left(g_{1} \otimes g_{2}\right):=\mathbf{m} \exp \left(\sum_{m, n \geq 1} t_{m n} \partial_{t_{m}} \otimes \partial_{t_{n}}\right) g_{1} \otimes g_{2},
$$

where no restriction is imposed on the parameters $t_{m n}$. The product $*_{2}$ may be regarded as being composed of Moyal deformations with parameters $\theta_{m n}=\left(t_{m n}-t_{n m}\right) / 2$ and 'trivial' deformations associated with the symmetric parts of the $t_{m n}$. The latter can be eliminated by means of an equivalence transformation (see [37], for example, and also [23]).19 We have the generalized Leibniz rule

$$
\partial_{t_{m n}}\left(g_{1} *_{2} g_{2}\right)=\left(\partial_{t_{m n}} g_{1}\right) *_{2} g_{2}+\left(\partial_{t_{m}} g_{1}\right) *_{2}\left(\partial_{t_{n}} g_{2}\right)+g_{1} *_{2}\left(\partial_{t_{m n}} g_{2}\right) \text {. }
$$

Now we proceed as in section 4

\footnotetext{
${ }^{18}$ The tensor product has to be taken over $\mathbb{K}\left[\left[\left\{t_{m n}\right\}\right]\right]$.

${ }^{19} \mathrm{~A}$ deformation with asymmetric $t_{m n}$ (which is equivalent to a Moyal deformation) appeared as follows in the context of integrable systems. The operation of taking the $\operatorname{symbol} \operatorname{sym}(P):=\sum_{-\infty<j \ll \infty} a_{j} p^{j}$ of a pseudo-differential operator $P=$ $\sum_{-\infty<j \ll \infty} a_{j} \partial_{x}^{j}$ has the homomorphism property $\operatorname{sym}\left(P P^{\prime}\right)=\operatorname{sym}(P) *_{\operatorname{sym}} \operatorname{sym}\left(P^{\prime}\right)$, where $a *_{\operatorname{sym}} b=\mathbf{m} \exp \left(\partial_{x} \otimes \partial_{p}\right) a \otimes b$ (see [4,7], for example). This allows to translate the Gelfand-Dickey formalism [36] of integrable systems into star product language. This is not, however, of relevance for the present work.
} 
Proposition 5 By use of (2.1), $Z_{t_{m n}}=0$ implies

$$
\phi_{t_{m n}}=-\phi_{t_{m}} *_{2}\left(R_{n}+Q_{n} \phi\right) .
$$

Under certain conditions (see the proof) also the converse holds.

Proof: In the following $*$ stands for $*_{2}$. Using $X_{t_{n}}=R_{n} X+Q_{n} Y$, we find

$$
\begin{aligned}
Y_{t_{m n}} & =(\phi * X)_{t_{m n}}=\phi_{t_{m n}} * X+\phi_{t_{m}} * X_{t_{n}}+\phi * X_{t_{m n}} \\
& =\left(\phi_{t_{m n}}+\phi_{t_{m}} *\left(R_{n}+Q_{n} \phi\right)\right) * X+\phi * X_{t_{m n}}
\end{aligned}
$$

If $Z_{t_{m n}}=0$, this implies (6.3). Conversely, if (6.3) holds, then we have $Y_{t_{m n}}=Y * C_{m n}$ with $C_{m n}=$ $X^{*-1} * X_{t_{m n}}$, hence $Z_{t_{m n}}=Z * C_{m n}$. By use of (2.1), the integrability conditions $Z_{t_{m n} t_{r}}=Z_{t_{r} t_{m n}}$ lead to $C_{m n, t_{r}}=0$. The remaining integrability conditions $Z_{t_{m n} t_{r s}}=Z_{t_{r s} t_{m n}}$ then state that the connection $C:=\sum_{m, n \geq 1} C_{m n} d t_{m n}$ has vanishing curvature. Provided that this implies the existence of a gauge transformation $Z^{\prime}=Z * G$ such that the transformed connection vanishes, we obtain $Z_{t_{m n}}^{\prime}=0$.

Translating (6.3) into the WNA language, using the rules of section 3 and $A \circ_{1} B=A *_{2} Q B$, we find the following deformation equations

$$
f_{t_{m n}}=f_{t_{m}} \circ_{n} f \quad m, n=1,2, \ldots .
$$

By use of $f_{t_{m}}=f \circ_{m} f$, this becomes a system of ordinary differential equations,

$$
f_{t_{m n}}=\left(f \circ_{m} f\right) \circ_{n} f=f \circ_{m}\left(f \circ_{n} f\right)-f \circ_{m+n} f .
$$

The commutativity condition for an equation of the system (6.5), with fixed $m$ and $n$, and $f_{t_{r}}=f \circ_{r} f$ also requires that $f_{t_{m}}=f \circ_{m} f$ and $f_{t_{n}}=f \circ_{n} f$ are satisfied. Furthermore, in order that two flows of the system (6.5) commute, also the associated four equations of the hierarchy (3.6) are needed. The origin of this conditional commutativity has already been identified in section 4

Remark. Assuming $Z_{t_{m n}}=H^{m+n} Z$ instead of $Z_{t_{m n}}=0$, a straightforward calculation leads to $f_{t_{m n}}=$ $f \circ_{m}\left(f \circ_{n} f\right)$ instead of (6.5). The origin of this alternative can be understood as follows. In general, $Z_{0}$ in the solution (2.5) to the linear system (2.1) may depend on the deformation parameters. Choosing the solution $Z^{\prime}=e^{t_{1} H_{2}} *_{2} e^{t_{2} H^{2}} *_{2} \cdots Z_{0}$, where $\left(Z_{0}\right)_{t_{m n}}=0$, this can be evaluated to $Z^{\prime}=e^{\xi(H)} Z_{0}^{\prime}$, where the dependence on deformation parameters is absorbed in $Z_{0}^{\prime}$. But now $Z^{\prime}$ solves $Z_{t_{m n}}=H^{m+n} Z$. The second equality of (6.5) shows that the new deformation equation differs from the old by the rhs of an equation of the hierarchy (3.6).

We can proceed to a further deformation by setting 20

$$
g_{1} *_{3} g_{2}:=\mathbf{m}_{3}\left(g_{1} \otimes g_{2}\right):=\mathbf{m}_{2} \exp \left(\sum_{m, n, r \geq 1} t_{m n r}\left(\partial_{t_{m n}} \otimes \partial_{t_{r}}+\partial_{t_{m}} \otimes \partial_{t_{n r}}\right)\right) g_{1} \otimes g_{2},
$$

with new deformation parameters $t_{m n r}$. This involves deformations not only with respect to the independent variables $t_{m}$, but also the previously introduced deformation parameters $t_{r s}$. It indeed defines an associative product 21 There is a corresponding generalized Leibniz rule,

$$
\partial_{t_{m n r}}\left(g_{1} *_{3} g_{2}\right)=\left(\partial_{t_{m n r}} g_{1}\right) *_{3} g_{2}+\left(\partial_{t_{m n}} g_{1}\right) *_{3}\left(\partial_{t_{r}} g_{2}\right)+\left(\partial_{t_{m}} g_{1}\right) *_{3}\left(\partial_{t_{n r}} g_{2}\right)+g_{1} *_{3}\left(\partial_{t_{m n r}} g_{2}\right)
$$

\footnotetext{
${ }^{20}$ Here the tensor product has to be taken over $\mathbb{K}\left[\left[\left\{t_{m n r}\right\}\right]\right]$. But before application of $\mathbf{m}_{2}$, it has to be projected to a tensor product over $\mathbb{K}\left[\left[\left\{t_{k l}, t_{m n r}\right\}\right]\right]$ (and $\mathbf{m}_{2}$ then has to be built with the latter). Our notation is a bit sloppy in this respect. But this should not really cause problems in actual calculations.

${ }^{21}$ The associativity can be checked as follows. First one verifies the identities $P\left(\mathbf{m}_{2} \otimes \mathrm{id}\right)=\left(\mathbf{m}_{2} \otimes \mathrm{id}\right)\left(P_{13}+P_{23}+\right.$ $\left.\sum t_{m n r} \partial_{t_{m}} \otimes \partial_{t_{n}} \otimes \partial_{t_{r}}\right)$ and $P\left(\mathrm{id} \otimes \mathbf{m}_{2}\right)=\left(\mathrm{id} \otimes \mathbf{m}_{2}\right)\left(P_{12}+P_{13}+\sum t_{m n r} \partial_{t_{m}} \otimes \partial_{t_{n}} \otimes \partial_{t_{r}}\right)$ where $P:=\sum t_{m n r}\left(\partial_{t_{m n}} \otimes\right.$ $\partial_{t_{r}}+\partial_{t_{m}} \otimes \partial_{t_{n r}}$ ), and, e.g., $P_{13}$ is given by $P$ acting on the first and third component of a threefold tensor product (and as identity on the second). Writing $\mathbf{m}_{3}\left(\mathbf{m}_{3} \otimes \mathrm{id}\right)=\mathbf{m}_{2} e^{P}\left(\mathbf{m}_{2} \otimes \mathrm{id}\right)\left(e^{P} \otimes \mathrm{id}\right)$, using the first identity, then the associativity condition $\mathbf{m}_{2}\left(\mathbf{m}_{2} \otimes\right.$ id $)=\mathbf{m}_{2}\left(\right.$ id $\left.\otimes \mathbf{m}_{2}\right)$ for $\mathbf{m}_{2}$, and in the next step the second identity, we obtain the associativity condition $\mathbf{m}_{3}\left(\mathbf{m}_{3} \otimes \mathrm{id}\right)=\mathbf{m}_{3}\left(\mathrm{id} \otimes \mathbf{m}_{3}\right)$.
} 
Assuming $Z_{t_{m n r}}=0$ in addition to the linear system (2.1) and $Z_{t_{m n}}=0$, we are led to the deformation equations

$$
\phi_{t_{m n r}}=-\phi_{t_{m n}} *_{3}\left(R_{r}+Q_{r} \phi\right),
$$

for which we find the following WNA version,

$$
\begin{aligned}
f_{t_{m n r}} & =f_{t_{m n}} \circ_{r} f=\left(f_{t_{m}} \circ_{n} f\right) \circ_{r} f=\left(\left(f \circ_{m} f\right) \circ_{n} f\right) \circ_{r} f \\
& =f \circ_{m}\left(f \circ_{n}\left(f \circ_{r} f\right)\right)-f \circ_{m+n}\left(f \circ_{r} f\right)-f \circ_{m}\left(f \circ_{n+r} f\right)+f \circ_{m+n+r} f,
\end{aligned}
$$

where now $A \circ_{1} B=A * 3 Q B$. Again, these flows commute with all other flows and among themselves conditionally, which now involves equations from (3.6) as well as from (6.5).

Remark. If $g_{1}, g_{2}, g_{3}$ do not depend on the parameters $t_{k l}$ and $t_{m n r}$, then we have

$$
\left(g_{1} *_{3} g_{2} *_{3} g_{3}\right)_{t_{m n r}}=g_{1, t_{m}} *_{3} g_{2, t_{n}} *_{3} g_{3, t_{r}} .
$$

As a consequence,

$$
\left\{g_{1}, g_{2}, g_{3}\right\}_{*_{3}}=\sum_{\sigma \in S_{3}} \operatorname{sign}(\sigma) g_{\sigma(1)} *_{3} g_{\sigma(2)} *_{3} g_{\sigma(3)}
$$

yields an apparently new deformation quantization of the canonical Nambu bracket of order 3 (see [38-42], for example). See also [43] for Nambu brackets in the context of integrable systems.

The above procedure can be continued by setting 22

$$
\mathbf{m}_{n+1}=\mathbf{m}_{n} \exp \left(\sum_{m_{1}, \ldots, m_{n+1} \geq 1} t_{m_{1} \ldots m_{n+1}} \sum_{r=1}^{n} \partial_{t_{m_{1} \ldots m_{r}}} \otimes \partial_{t_{m_{r+1} \ldots m_{n+1}}}\right) \quad n=1,2, \ldots
$$

It is not difficult to verify that these products are indeed associative (see also [23], appendix C).

Proposition 6 The linear system (2.1) supplemented by $Z_{t_{m_{1} \ldots m_{k}}}=0,2 \leq k \leq n$, implies the Riccati system $\phi_{t_{r}}=S_{r}+L_{r} \phi-\phi R_{r}-\phi *_{n} Q_{r} \phi, r=1,2, \ldots$, supplemented by

$$
\phi_{t_{m_{1} \ldots m_{k}}}=-\phi_{t_{m_{1} \ldots m_{k-1}}} *_{n}\left(R_{m_{k}}+Q_{m_{k}} \phi\right) \quad k=2, \ldots, n
$$

(where $\left.m_{1}, \ldots, m_{k}=1,2, \ldots\right)$.

Proof: This is a straightforward generalization of the proof of proposition 5

The extended Riccati system in the last proposition translates into

$$
f_{t_{m_{1} \ldots m_{k}}}=e_{\left(m_{1}, \ldots, m_{k}\right)} \quad k=1, \ldots, n,
$$

where

$$
e_{\left(m_{1}, \ldots, m_{k}\right)}:=R_{f}^{\left(m_{k}\right)} \cdots R_{f}^{\left(m_{1}\right)}(f)
$$

and $R_{f}^{(m)}(a):=a \circ_{m} f$, hence $e_{\left(m_{1}, \ldots, m_{k}\right)} \circ_{m_{k+1}} f=e_{\left(m_{1}, \ldots, m_{k+1}\right)}$. The products $\circ_{m}$ involve the $*_{n}$ product. It is convenient to replace it by the $n \rightarrow \infty$ limit of the $*_{n}$ products and this will be done in the following.

\footnotetext{
${ }^{22}$ The remarks in footnote 20 apply correspondingly here. For an alternative but equivalent definition of these products see [23]. Exploiting equivalence transformations of star products (see [37], for example), some parameters appear to be redundant. However, also deformation parameters which are redundant in this sense can play a non-trivial role e.g. as evolution variables of nonlinear integrable equations, see section 7
} 
Lemma 3 Let $f_{t_{m_{1} \ldots m_{k}}} \in \mathbb{A}^{\prime}$ for $k=1, \ldots, r$. The partial derivative with respect to $t_{m_{1} \ldots m_{r}}$ then satisfies the generalized Leibniz rule

$$
\begin{aligned}
\left(a \circ_{n} b\right)_{t_{m_{1} \cdots m_{r}}} & =\sum_{k=0}^{r} a_{t_{m_{1} \cdots m_{k}}} \circ_{n} b_{t_{m_{k+1} \cdots m_{r}}} \\
& =a \circ_{n} b_{t_{m_{1} \cdots m_{r}}}+\sum_{k=1}^{r-1} a_{t_{m_{1} \cdots m_{k}}} \circ_{n} b_{t_{m_{k+1} \cdots m_{r}}}+a_{t_{m_{1} \cdots m_{r}}} \circ_{n} b
\end{aligned}
$$

with respect to all products $\circ_{n}, n=1,2, \ldots$, defined by (3.5) in terms of $f$.

Proof: This holds for $n=1$ (cf. also [23], appendix C). The general statement is then obtained by induction as follows. By use of (3.5) and the induction hypothesis, we find

$$
\begin{aligned}
\left(a \circ_{n+1} b\right)_{t_{m_{1} \cdots m_{r}}}= & \sum_{0 \leq k \leq l \leq r}\left(a_{t_{m_{1} \cdots m_{k}}} \circ_{1}\left(f_{t_{m_{k+1} \cdots m_{l}} \circ_{n}} b_{t_{m_{l+1} \cdots m_{r}}}\right)\right. \\
& \left.-\left(a_{t_{m_{1} \cdots m_{k}}} \circ_{1} f_{t_{m_{k+1} \cdots m_{l}}}\right) \circ_{n} b_{t_{m_{l+1} \cdots m_{r}}}\right) .
\end{aligned}
$$

Because of our assumption and the WNA property of the products (see proposition 3.1 in [29]), only the terms with $k=l$ survive. Since $f_{t_{m_{k+1} \cdots m_{k}}}$ has to be read as $f$, this leads to

$$
\begin{aligned}
\left(a \circ_{n+1} b\right)_{t_{m_{1} \cdots m_{r}}} & =\sum_{0 \leq k \leq r}\left(a_{t_{m_{1} \cdots m_{k}}} \circ_{1}\left(f \circ_{n} b_{t_{m_{k+1} \cdots m_{r}}}\right)-\left(a_{t_{m_{1} \cdots m_{k}}} \circ_{1} f\right) \circ_{n} b_{t_{m_{k+1} \cdots m_{r}}}\right) \\
& =\sum_{0 \leq k \leq r} a_{t_{m_{1} \cdots m_{k}}} \circ_{n+1} b_{t_{m_{k+1} \cdots m_{r}}} .
\end{aligned}
$$

Proposition 7 The flows given by

$$
f_{t_{m_{1} \ldots m_{n}}}=e_{\left(m_{1}, \ldots, m_{n}\right)} \quad n=1,2, \ldots, \quad m_{k} \in \mathbb{N}
$$

commute with each other.

Proof: We use induction. For $n=1$ the statement is contained in theorem 1 Using $e_{\left(m_{1}, \ldots, m_{r+1}\right)}=$ $e_{\left(m_{1}, \ldots, m_{r}\right)}{ }^{\circ} m_{r+1} f$ and the preceding lemma (the assumptions of which are satisfied as a consequence of (6.16)), we obtain

$$
\begin{aligned}
& f_{t_{m_{1} \cdots m_{r+1}} t_{n_{1} \cdots n_{s+1}}}=e_{\left(m_{1}, \ldots, m_{r+1}\right), t_{n_{1} \cdots n_{s+1}}} \\
= & e_{\left(m_{1}, \ldots, m_{r}\right), t_{n_{1} \cdots n_{s+1}}} \circ_{m_{r+1}} f+\sum_{k=0}^{s} e_{\left(m_{1}, \ldots, m_{r}\right), t_{n_{1} \cdots n_{k}}} \circ_{m_{r+1}} e_{\left(n_{k+1}, \ldots, n_{s+1}\right)},
\end{aligned}
$$

where we made use of some 'lower' equations of the system (6.16). By induction hypothesis, the first summand of the last expression is equal to

$$
\begin{aligned}
& e_{\left(n_{1}, \ldots, n_{s+1}\right), t_{m_{1} \cdots m_{r}}} \circ_{m_{r+1}} f \\
= & \left(e_{\left(n_{1}, \ldots, n_{s}\right), t_{m_{1} \cdots m_{r}}} \circ_{n_{s+1}} f+\sum_{k=0}^{r-1} e_{\left(n_{1}, \ldots, n_{s}\right), t_{m_{1} \cdots m_{k}}} \circ_{n_{s+1}} e_{\left(m_{k+1}, \ldots, m_{r}\right)}\right) \circ_{m_{r+1}} f
\end{aligned}
$$




$$
\begin{aligned}
= & \left(e_{\left(n_{1}, \ldots, n_{s}\right), t_{m_{1} \cdots m_{r}}} \circ_{n_{s+1}} f\right) \circ_{m_{r+1}} f+\sum_{k=0}^{r-1} e_{\left(n_{1}, \ldots, n_{s}\right), t_{m_{1} \cdots m_{k}}} \circ_{n_{s+1}} e_{\left(m_{k+1}, \ldots, m_{r+1}\right)} \\
= & \left(e_{\left(n_{1}, \ldots, n_{s}\right), t_{m_{1} \cdots m_{r}}} \circ_{n_{s+1}} f\right) \circ_{m_{r+1}} f-e_{\left(n_{1}, \ldots, n_{s}\right), t_{m_{1} \cdots m_{r}}} \circ_{n_{s+1}}\left(f \circ_{m_{r+1}} f\right) \\
& +\sum_{k=0}^{r} e_{\left(n_{1}, \ldots, n_{s}\right), t_{m_{1} \cdots m_{k}}} \circ_{n_{s+1}} e_{\left(m_{k+1}, \ldots, m_{r+1}\right)} \\
= & -e_{\left(n_{1}, \ldots, n_{s}\right), t_{m_{1} \cdots m_{r}}} \circ_{\left(m_{r+1}+n_{s+1}\right)} f+\sum_{k=0}^{r} e_{\left(n_{1}, \ldots, n_{s}\right), t_{m_{1} \cdots m_{k}}} \circ_{n_{s+1}} e_{\left(m_{k+1}, \ldots, m_{r+1}\right)},
\end{aligned}
$$

by use of proposition 3.3 in [29]. Inserting this in the above expression for $f_{t_{m_{1} \cdots m_{r+1}} t_{n_{1} \cdots n_{s+1}}}$, and applying the induction hypothesis to $e_{\left(n_{1}, \ldots, n_{s}\right), t_{m_{1} \cdots m_{r}}}$, results in an expression which is symmetric in $m$ 's and $n$ 's. This implies our assertion.

For $n=1$, (6.16) is the hierarchy (3.6). Whereas any two flows of the latter system commute with each other without further conditions, the commutativity of two flows of the extended system (6.16) on the $n$th level, $n>1$, also requires some flow equations on lower levels, as explained above. The structure of the proof of the last proposition clearly displays this fact. Because of this conditional commutativity, (6.16) is not quite a hierarchy in the familiar sense, though a natural generalization, which obviously preserves the integrability of the hierarchy.

By elimination of $L, R, S$ from (6.13) (using also the Riccati system (2.12)), respectively elimination of bare $f$ 's from (6.16), one obtains deformation equations of the deformed (matrix) pKP hierarchy by an extension of the methods in section 5] (see also [23] for a different approach). Again, these deformation equations extend the deformed $\mathrm{pKP}$ hierarchy.

Remark. We know from [29] that the monomial $e_{\left(m_{1}, \ldots, m_{n}\right)}$ is related to a basis element of the space of quasi-symmetric functions (see [44-46], for example) in commuting variables $k_{1}, k_{2}, \ldots, k_{N}$, given by

$$
M_{\left(m_{1}, \ldots, m_{n}\right)}=\sum_{1 \leq r_{1}<r_{2}<\cdots<r_{n} \leq N} k_{r_{1}}^{m_{1}} k_{r_{2}}^{m_{2}} \cdots k_{r_{n}}^{m_{n}} .
$$

The relation of the more general deformations, considered in this section, with quasi-symmetric functions emerges as follows on a simpler level. Products of the form

$$
e^{\xi\left(k_{1}\right)} *_{n} e^{\xi\left(k_{2}\right)} *_{n} \cdots *_{n} e^{\xi\left(k_{N}\right)}=: e^{\Xi\left(k_{1}, \ldots, k_{N}\right)}
$$

with $\xi(k)=\sum_{n \geq 1} t_{n} k^{n}$ are constituents of deformed soliton solutions as obtained in [23]. We find 23

$$
\Xi\left(k_{1}, \ldots, k_{N}\right)=\sum_{r=1}^{n+1} \sum_{m_{1}, \ldots, m_{r} \geq 1} t_{m_{1} \ldots m_{r}} M_{\left(m_{1}, \ldots, m_{r}\right)} .
$$

\section{Via deformation to KP}

Let us start with an associative algebra where the elements depend smoothly on (only) $t_{1}$. The deformations considered in section 6 then reduce to those associated with the deformation parameters

$$
t_{1^{k}}:=t_{1 \ldots 1} \quad k=2,3, \ldots .
$$

\footnotetext{
${ }^{23}$ Note that $M_{\left(m_{1}, \ldots, m_{r}\right)}=0$ if $r>N$. See also section 8.1 of [23].
} 
Let $*$ denote the $n \rightarrow \infty$ limit of the corresponding products $*_{n}$. The set of derivatives $\left\{\partial_{t_{1 k}}\right\}_{k \in \mathbb{N}}$ is a Hasse-Schmidt derivation of the product $*$, i.e. they satisfy the generalized derivation rule

$$
\left(g_{1} * g_{2}\right)_{t_{1 k}}=\sum_{j=0}^{k} g_{1, t_{1 j}} * g_{2, t_{1 k-j}}=g_{1} * g_{2, t_{1} k}+\sum_{j=1}^{k-1} g_{1, t_{1 j}} * g_{2, t_{1 k-j}}+g_{1, t_{1 k}} * g_{2} .
$$

Given commuting (ordinary) derivations $\tilde{\partial}_{k}, k=1,2, \ldots$, the set $\left\{(-1)^{k} \mathbf{p}_{k}(-\tilde{\partial})\right\}_{k \in \mathbb{N}}$, with the elementary Schur polynomials $\mathbf{p}_{k}$ and $\tilde{\partial}=\left(\widetilde{\partial}_{t_{1}}, \frac{1}{2} \widetilde{\partial}_{t_{2}}, \frac{1}{3} \widetilde{\partial}_{t_{3}}, \ldots\right)$, also yields a Hasse-Schmidt derivation. This observation allows us to define derivations $\partial_{k}, k=1,2, \ldots$, by setting

$$
\partial_{t_{1 k}}=:(-1)^{k} \mathbf{p}_{k}(-\tilde{\tilde{\partial}}) \quad k=1,2, \ldots
$$

In particular, we have $\partial_{1}=\partial_{t_{1}}$ and $\partial_{2}=\partial_{t_{1}}^{2}-2 \partial_{t_{11}}$.

Now, what has all this to do with KP? According to our general strategy, we should start from the linear equation

$$
Z_{t_{1}}=H Z
$$

and assume $Z$ to be independent of the deformation parameters, i.e. 24

$$
Z_{t^{n}}=0 \quad n=2,3, \ldots .
$$

By use of the last condition, (7.2), and $X_{t_{1}}=R X+Q Y$ (which follows from (7.4) with the decomposition (2.2), we obtain

$$
\phi_{t_{1^{n+1}}}=-\sum_{k=0}^{n} \phi_{t_{1^{k}}} * X_{t_{1^{n-k}}} * X^{*-1}=-\phi_{t_{1^{n}}} * X_{t_{1}} * X^{*-1}=-\phi_{t_{1^{n}}} *(Q \phi+R) .
$$

This in turn leads to

$$
\phi_{t_{1^{n+1}} t_{1} m}=-\phi_{t_{1^{n} t_{1} m}} *(Q \phi+R)-\sum_{k=0}^{m-1} \phi_{t_{1^{n} t_{1^{k}}}} * Q \phi_{t_{1^{m-k}}},
$$

and consequently, by elimination of $R$,

$$
\phi_{t_{1^{n+1}} t_{1} m}-\phi_{t_{1^{m+1}} t_{1^{n}}}=\sum_{k=0}^{n-1} \phi_{t_{1^{m} t_{1^{k}}}} * Q \phi_{t_{1^{n-k}}}-\sum_{k=0}^{m-1} \phi_{t_{1^{n} t_{1^{k}}}} * Q \phi_{t_{1^{m-k}}},
$$

which by use of (7.3) results in

$$
\begin{aligned}
& \mathbf{p}_{n+1}(-\tilde{\tilde{\partial}}) \mathbf{p}_{m}(-\tilde{\tilde{\partial}}) \phi-\mathbf{p}_{m+1}(-\tilde{\widetilde{\partial}}) \mathbf{p}_{n}(-\tilde{\widetilde{\partial}}) \phi \\
& =\sum_{k=0}^{m-1} \mathbf{p}_{k}(-\tilde{\tilde{\delta}}) \mathbf{p}_{n}(-\tilde{\tilde{\delta}}) \phi * Q \mathbf{p}_{m-k}(-\tilde{\tilde{\partial}}) \phi-\sum_{k=0}^{n-1} \mathbf{p}_{k}(-\tilde{\tilde{\delta}}) \mathbf{p}_{m}(-\tilde{\tilde{\delta}}) \phi * Q \mathbf{p}_{n-k}(-\tilde{\tilde{\delta}}) \phi \text {, }
\end{aligned}
$$

where $m, n=1,2, \ldots$. Abstracting from the origin of $\phi$ and regarding this as a system imposed on some $\phi\left(t_{1}, t_{11}, \ldots\right)$, it resembles a known formulation of the pKP hierarchy [29,47], though with the deformed

\footnotetext{
${ }^{24}$ By use of (7.3), the system (7.5) is equivalent to $\partial_{n}(Z)=\partial_{t_{1}}^{n}(Z), n=2,3, \ldots$. Via $\partial_{n} \mapsto \partial_{t_{n}}$, which is the effect of the homomorphism ^ introduced below, this becomes the linear heat hierarchy $Z_{t_{n}}=\partial_{t_{1}}^{n}(Z)$.
} 
product and with partial derivatives with respect to variables $t_{2}, t_{3}, \ldots$ replaced by $\partial_{k}, k=2,3, \ldots$. Since the latter are derivations of the product $*$, the operator defined by

$$
\hat{g}:=\exp \left(\sum_{k=2}^{n} t_{k} \mho_{k}\right) g
$$

is a homomorphism, which satisfies

$$
\hat{g}_{t_{k}}=\widehat{\partial_{k}(g)} \text {. }
$$

Applying this operator to (7.9), and finally setting all deformation parameters to zero, we obtain for

$$
\hat{\phi}:=\left.\exp \left(\sum_{k=2}^{n} t_{k} \partial_{k}\right) \phi\right|_{t_{1 k}=0, k>1}
$$

the pKP hierarchy equations

$$
\begin{aligned}
& \mathbf{p}_{n+1}(-\tilde{\partial}) \mathbf{p}_{m}(-\tilde{\partial}) \hat{\phi}-\mathbf{p}_{m+1}(-\tilde{\partial}) \mathbf{p}_{n}(-\tilde{\partial}) \hat{\phi} \\
= & \sum_{k=0}^{m-1} \mathbf{p}_{k}(-\tilde{\partial}) \mathbf{p}_{n}(-\tilde{\partial}) \hat{\phi} Q \mathbf{p}_{m-k}(-\tilde{\partial}) \hat{\phi}-\sum_{k=0}^{n-1} \mathbf{p}_{k}(-\tilde{\partial}) \mathbf{p}_{m}(-\tilde{\partial}) \hat{\phi} Q \mathbf{p}_{n-k}(-\tilde{\partial}) \hat{\phi}
\end{aligned}
$$

Any solution $\phi\left(t_{1}, t_{11}, \ldots\right)$ of (7.8) thus yields, via (7.12), a solution of the pKP hierarchy (in the matrix algebra with product modified by $Q$ ).

The way towards solutions of the pKP hierarchy described above is surprisingly simple. We have to write down the solution of the single linear ordinary differential equation (7.4), which is a matrix of functions of the variable $t_{1}$. All the other variables, the evolution parameters of the hierarchy, emerge in the pKP solution $\phi$ as deformation parameters through the product!

Example. Let $S=0$ and $Q=R K-K L$ with an $N \times M$ matrix $K$ (cf. (2.16)). Then the solution of the linear equation (7.4) takes the form

$$
Z=e^{t_{1} H} Z_{0}=\left(\begin{array}{c}
e^{t_{1} R} X_{0}+\left(e^{t_{1} R} K-K e^{t_{1} L}\right) Y_{0} \\
e^{t_{1} L} Y_{0}
\end{array}\right)
$$

which leads to the solution 25

$$
\phi=e^{t_{1} L} \phi_{0} *\left(e^{t_{1} R}\left(I_{N}+K \phi_{0}\right)-K e^{t_{1} L} \phi_{0}\right)^{*-1},
$$

of (7.8), where $\phi_{0}:=Y_{0} X_{0}^{-1}$. This expression is solely built from functions of $t_{1}$ only. The additional variables enter via the deformed product. Using the homomorphism property of the operation ${ }^{\wedge}$, we recover the solution (2.18) of the pKP hierarchy.

Remark. The deformation considered in this section is trivial in the sense that it can be transformed away by an equivalence transformation of star products. This will be demonstrated in the following. Consider the product

$$
g_{1} \star_{n} g_{2}:=\mathcal{D}_{n}^{-1}\left(\mathcal{D}_{n}\left(g_{1}\right) *_{n-1} \mathcal{D}_{n}\left(g_{2}\right)\right),
$$

\footnotetext{
${ }^{25}$ Note that the $*$-inverse of a function of $t_{1}$ depends on all deformation parameters. For example, the inverse of $e^{t_{1} L}$ is given by $e^{-t_{1} L+t_{11} L^{2}-t_{111} L^{3}+\ldots}$.
} 
with

$$
\mathcal{D}_{n}=e^{-t_{1} \Upsilon} \Upsilon_{n},
$$

where $\Upsilon_{n}$ is an operator that commutes with $t_{1^{n}}$. Then

$$
\left(g_{1} \star_{n} g_{2}\right)_{t_{1} n}=\Upsilon_{n}\left(g_{1} \star_{n} g_{2}\right)-\Upsilon_{n}\left(g_{1}\right) \star_{n} g_{2}-g_{1} \star_{n} \Upsilon_{n}\left(g_{2}\right)+g_{1, t_{1} n} \star_{n} g_{2}+g_{1} \star_{n} g_{2, t_{1} n}
$$

Now let us choose

$$
\Upsilon_{n}=(-1)^{n} \mathbf{p}_{n}(-\tilde{\tilde{\partial}})+\frac{1}{n}(-1)^{n} \partial_{n}
$$

Here the last term cancels a corresponding term in $(-1)^{n} \mathbf{p}_{n}(-\tilde{\tilde{\delta}})=\partial_{t_{1} n}$, so that the remaining part can be expressed in terms of partial derivatives with respect to $\partial_{t_{1} k}$ with $k<n$ only. Using the derivation property of $\check{\partial}_{n}$, we find

$$
\begin{aligned}
\left(g_{1} \star_{n} g_{2}\right)_{t_{1 n}}= & (-1)^{n} \mathbf{p}_{n}(-\tilde{\delta})\left(g_{1} \star_{n} g_{2}\right)-(-1)^{n} \mathbf{p}_{n}(-\tilde{\delta})\left(g_{1}\right) \star_{n} g_{2}-g_{1} \star_{n}(-1)^{n} \mathbf{p}_{n}(-\tilde{\delta})\left(g_{2}\right) \\
& +g_{1, t_{1} n} \star_{n} g_{2}+g_{1} \star_{n} g_{2, t_{1} n} \\
= & \sum_{k=0}^{n} \partial_{t_{1 k}}\left(g_{1}\right) \star_{n} \partial_{t_{1^{n-k}}}\left(g_{2}\right)
\end{aligned}
$$

with the help of the (Hasse-Schmidt) generalized derivation property of $(-1)^{n} \mathbf{p}_{n}(-\tilde{\tilde{\partial}})$, and (7.3). This is precisely the expression (7.2) with products $\star_{n}$ and $*_{n}$ exchanged, and this also holds for the iterated derivatives. Since these expressions coincide for both products at $t_{1^{n}}=0$, we conclude that the two products are the same. We have shown that $*_{n}$ is obtained from $*_{n-1}$ by an equivalence transformation of star products, in the case where only the deformation parameters $t_{1^{n}}, n>1$, are switched on.

Remark. With the (restricted) deformation considered in this section, we have

$$
e^{t_{1} k_{1}} * \cdots * e^{t_{1} k_{N}}=e^{\Xi\left(k_{1}, \ldots, k_{N}\right)}
$$

with

$$
\Xi\left(k_{1}, \ldots, k_{N}\right)=t_{1} M_{(1)}+t_{11} M_{(1,1)}+\ldots+t_{1^{N}} M_{\left(1^{N}\right)}
$$

(see the last remark in section 6). The expressions $M_{\left(1^{k}\right)}, k=1, \ldots, N$, are the elementary symmetric functions in $k_{1}, \ldots, k_{N}$. These are related to the power symmetric functions $M_{(k)}$ through the relations

$$
M_{\left(1^{n}\right)}=(-1)^{n} \mathbf{p}_{n}(-\tilde{M}) \quad n=1,2, \ldots, \quad \tilde{M}:=\left(M_{(1)}, M_{(2)} / 2, M_{(3)} / 3, \ldots\right)
$$

(cf. [48], the second of equations (2.14') in chapter I). Thus, via the product $*$, elementary symmetric functions $M_{\left(1^{k}\right)}$ are introduced in our expressions. After use of the last relation to express the elementary symmetric functions in terms of power symmetric functions, the hat operator then generates the new coefficients $t_{2}, t_{3}, \ldots$ via

$$
\hat{\Xi}\left(k_{1}, \ldots, k_{N}\right)=\Xi\left(k_{1}, \ldots, k_{N}\right)+t_{2} M_{(2)}+\cdots+t_{N} M_{(N)} .
$$

Elementary symmetric functions no longer show up in the final expressions, after setting $t_{1^{k}}=0, k>1$. 


\section{Conclusions}

In the presence of a Moyal or (in the sense of section 6) generalized deformation, the (potential) KP hierarchy extends to a larger hierarchy which includes additional flows with the deformation parameters as new evolution variables. This observation has already been made in previous work (see [23], in particular). Here we presented a new and quite simple approach which also yields exact solutions.

As already pointed out in section 4 in this case we are actually dealing with a family of KP hierarchies, parametrized by deformation parameters of the product. From the analogy with Seiberg-Witten maps it should be obvious that a deformation equation does not generate a homomorphism of an algebra, but rather a homomorphism between different algebras (corresponding to different non-zero values of the deformation parameters). This is mirrored in the fact that the derivatives $\partial_{t_{m_{1} \ldots m_{r}}}, r>1$, are not derivations.

In [29] we proved that any solution of the hierarchy (3.6) of ordinary differential equations in any WNA algebra $\mathbb{A}$ determines a solution of the potential KP hierarchy in the middle nucleus $\mathbb{A}^{\prime}$ of $\mathbb{A}$. The present work extended the hierarchy (3.6) by corresponding ordinary differential equations in the deformation parameters.

Concerning the significance of an extended hierarchy, we already made some remarks in the introduction, but this work further adds to it. In section 7 we considered a sub-hierarchy of deformation equations which turned out to be the KP hierarchy in disguise. In this example, the KP hierarchy equations themselves emerge as deformation flow equations. Although the deformation is trivial in the sense that the deformation of the product can be eliminated by an equivalence transformation, the deformation flows act in a non-trivial way.

There is certainly much more to be discovered in the huge extended hierarchy with the general deformation treated in section 6 The fact that Moyal products exhibit structures of relevance in the theory of integrable systems (see [49,50], for example) provides us with further motivation. In fact, the results (of section 77 just mentioned demonstrate, in a completely independent way, the relevance of more general star products in this respect.

The FORM programs listed in the appendices should also be helpful to work out further examples beyond those presented in this work.

\section{Appendix A: Checking commutativity of flows with FORM in the KdV case}

In this appendix we list a simple FORM program $[25,26]$ which verifies that the flow of the Moyal-deformed (matrix) KdV equation (1.1) commutes with that of its deformation equation (1.2). The program can easily be adapted to other equations. FORM is especially helpful for such computations since by default it deals with noncommuting functions. In the following, an expression like $\mathrm{u}(3,2,1)$ stands for $u_{x x x t t \theta}$.

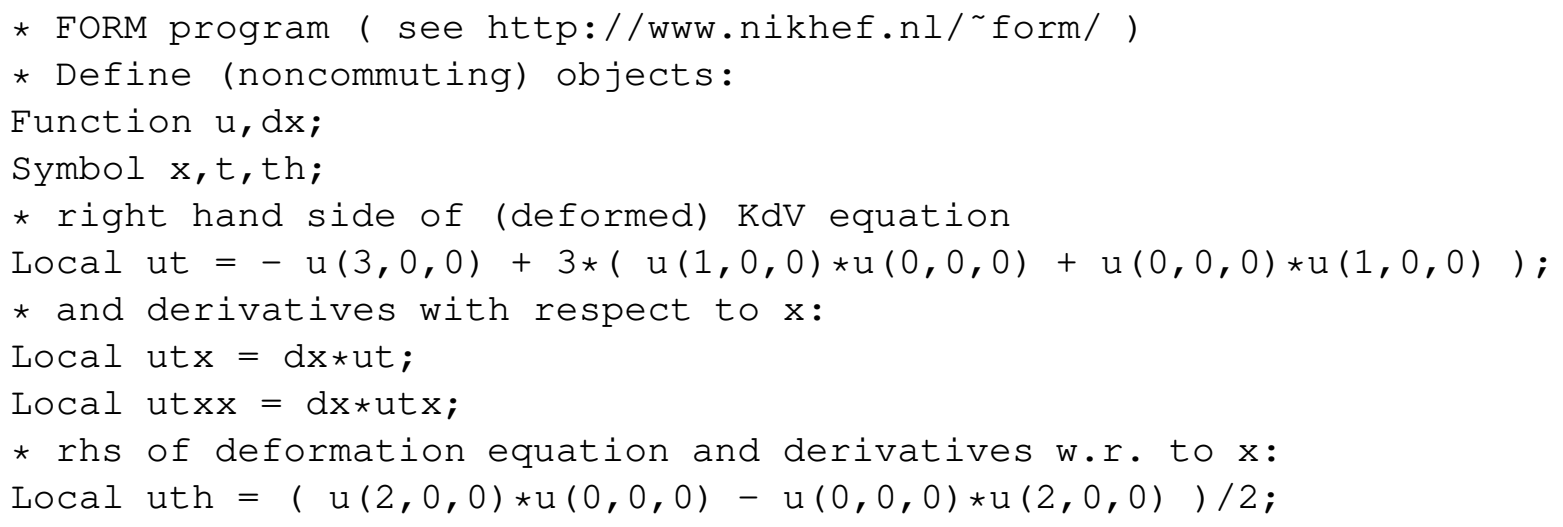




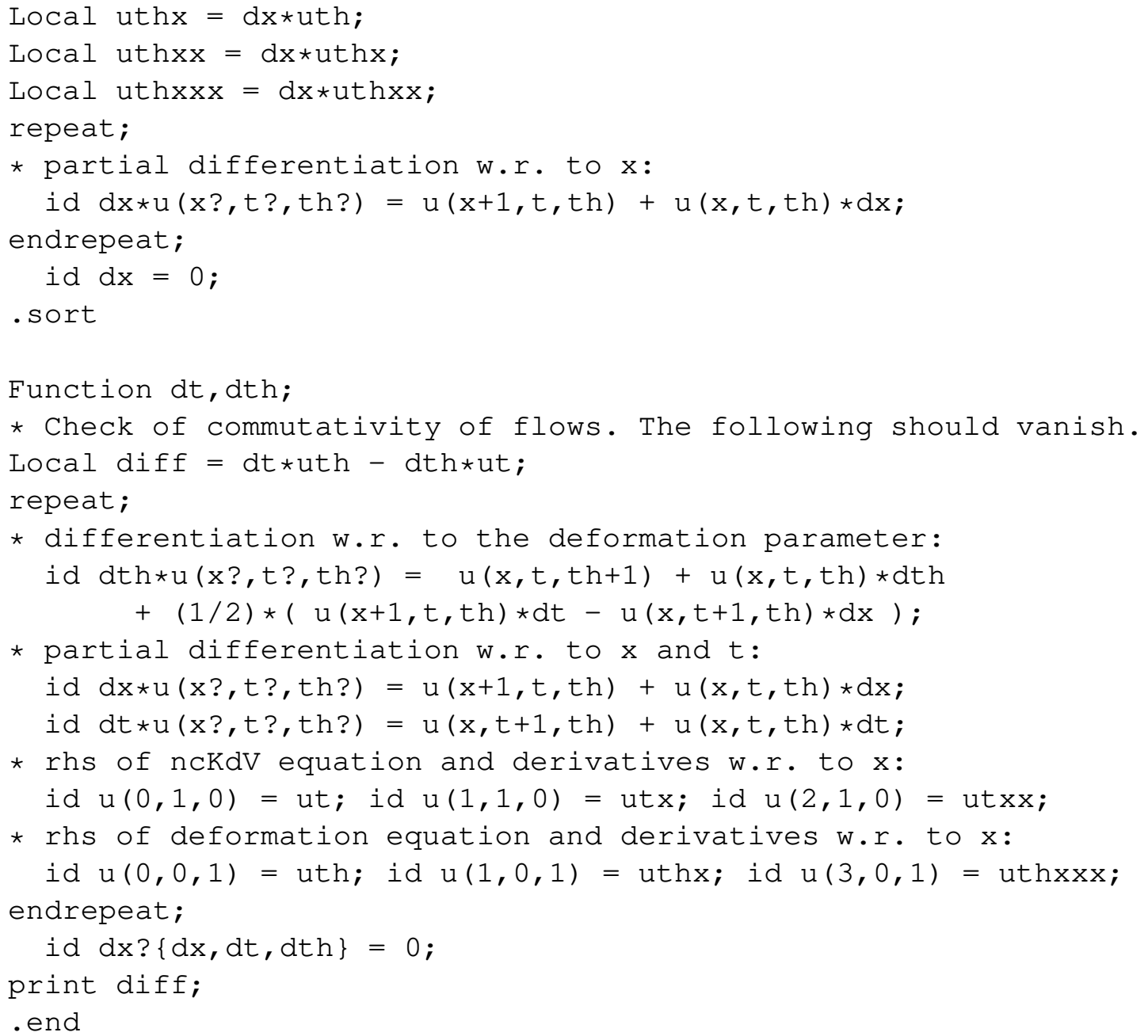

\section{Appendix B: Checking a deformation equation with FORM}

The following FORM program verifies that the right hand side of the deformation equation (4.6) with $m=1$ and $n=2$ becomes the right hand side of (4.8) by elimination of $L, R, S$, using the first three equations of the (Moyal-deformed) Riccati system (2.12). Here we set $x=t_{1}, y=t_{2}, t=t_{3}$.

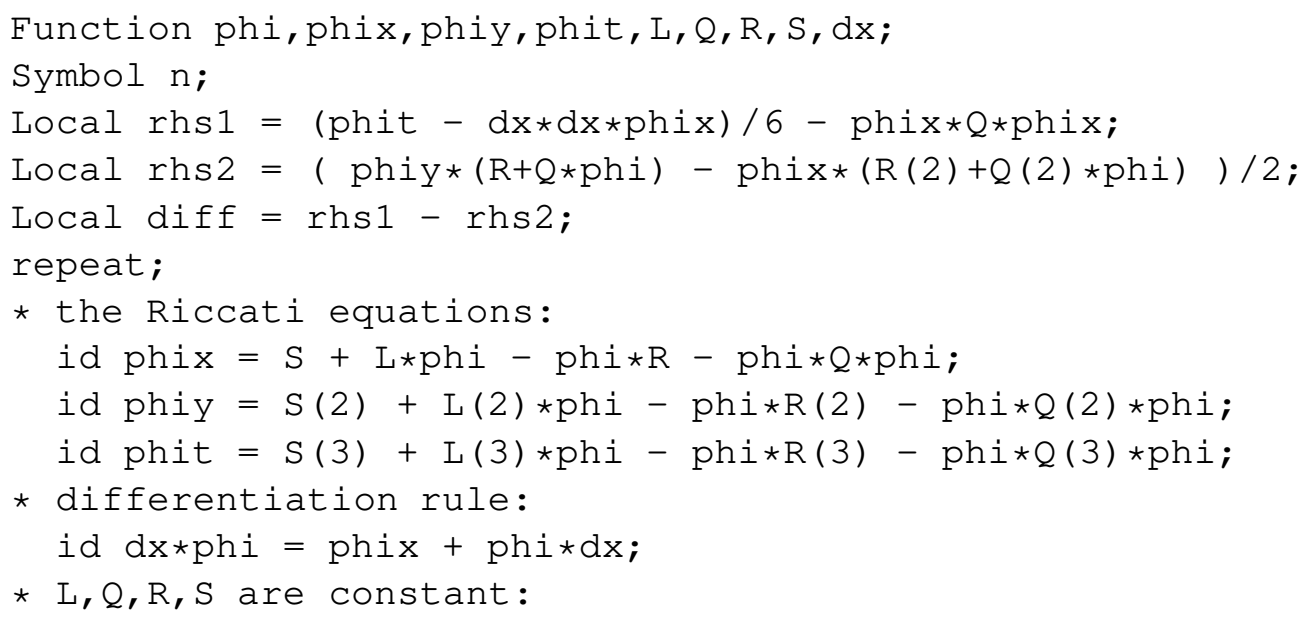




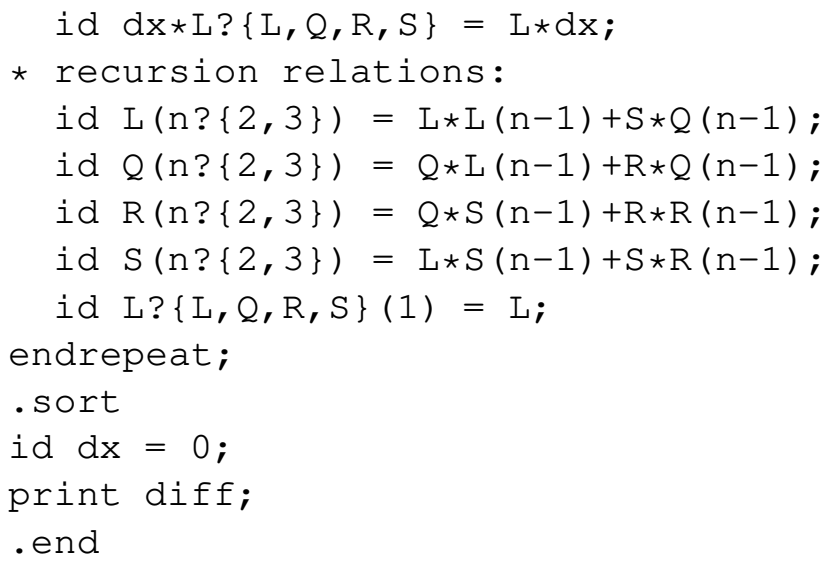

\section{References}

[1] Bayen F, Flato M, Fronsdal C, Lichnerowicz A, and Sternheimer D 1978 Deformation theory and quantization. I. Deformation of symplectic structures Ann. Phys. 111 61-110

[2] Douglas M R and Nekrasov N A 2001 Noncommutative field theory Rev. Mod. Phys. 73 977-1029

[3] Kupershmidt B A 1990 Quantizations and integrable systems Lett. Math. Phys. 20 19-31

[4] Figueroa-O'Farrill J M, Mas J and Ramos E 1993 A one-parameter family of Hamiltonian structures for the KP hierarchy and a continuous deformation of the nonlinear $W_{K P}$ algebra Commun. Math. Phys. 158 17-43

[5] Strachan I 1995 The Moyal bracket and the dispersionless limit of the KP hierarchy J. Phys. A: Math. Gen. 28 1967-1976

[6] Gawrylczyk J 1995 Relationship between the Moyal KP and the Sato KP hierarchies J. Phys. A: Math. Gen. 28 4381-4388

[7] Strachan I A B 1997 A geometry for multidimensional integrable systems J. Geom. Phys. 21 255-278

[8] Koikawa T 2001 Soliton equations extracted from the noncommutative zero-curvature equation Progr. Theor. Phys. 105 1045-1057

[9] Błaszak M and Szablikowski B M 2003 From dispersionless to soliton systems via Weyl-Moyal-like deformations J. Phys. A: Math. Gen. 36 12181-12203

[10] Carroll R 2003 Integrable systems as quantum mechanics Preprint quant-ph/0309159

[11] Takasaki K 1994 Nonabelian KP hierarchy with Moyal algebraic coefficients J. Geom. Phys. 14 332364

[12] Takasaki K 2001 Anti-self-dual Yang-Mills equations on noncommutative spacetime J. Geom. Phys. 37 291-306

[13] Wang N and Wadati M 2003 A new approach to noncommutative soliton equations J. Phys. Soc. Japan 72 3055-3062

[14] Lechtenfeld O and Popov A D 2004 Noncommutative monopoles and Riemann-Hilbert problems JHEP 01069 
[15] Hamanaka M 2005 Noncommutative solitons and integrable systems Preprint hep-th/0504001

[16] Dimakis A and Müller-Hoissen F 2000 The Korteweg-de-Vries equation on a noncommutative spacetime Phys. Lett. A 278 139-145

[17] Dimakis A and Müller-Hoissen F 2000 Moyal deformation, Seiberg-Witten map, and integrable models Lett. Math. Phys. 54 123-135

[18] Dimakis A and Müller-Hoissen F 2001 Noncommutative NLS equation Czech. J. Phys. 51 1285-1290

[19] Wang N 2004 Rescaling symmetry flow of the Kadomtsev-Petviashvili hierarchy Chin. Phys. Lett. 21 $2327-2329$

[20] Dimakis A and Müller-Hoissen F 2004 Extension of noncommutative soliton hierarchies J. Phys. A: Math. Gen. 37 4069-4084

[21] Dimakis A and Müller-Hoissen F 2004 Explorations of the extended ncKP hierarchy J. Phys. A: Math. Gen. 37 10899-10930

[22] Dimakis A and Müller-Hoissen F 2004 Extension of Moyal-deformed hierarchies of soliton equations XI International Conference Symmetry Methods in Physics ed Č Burdik, O Navrátil and S Pošta (Dubna: Joint Institute for Nuclear Research)

[23] Dimakis A and Müller-Hoissen F 2005 An algebraic scheme associated with the noncommutative KP hierarchy and some of its extensions J. Phys. A: Math. Gen. 38 5453-5505

[24] Seiberg N and Witten E 1999 String theory and noncommutative geometry JHEP 9909032

[25] Heck A 2000 FORM for Pedestrians (Amsterdam: NIKHEF)

[26] Vermaseren J A M 2002 FORM Reference Manual (Amsterdam: NIKHEF)

[27] Sato M and Sato Y 1982 Soliton equations as dynamical systems on infinite dimensional Grassmann manifold Nonlinear Partial Differential Equations in Applied Science ed H Fujita, P D Lax and G Strang (Amsterdam: North-Holland) pp 259-271

[28] Takasaki K 1989 Geometry of universal Grassmann manifold from algebraic point of view Rev. Math. Phys. 1 1-46

[29] Dimakis A and Müller-Hoissen F 2006 Nonassociativity and integrable hierarchies Preprint nlin.SI/0601001

[30] Dimakis A and Müller-Hoissen F 2007 Weakly nonassociative algebras, Riccati and KP hierarchies Preprint nlin.SI/0701010

[31] Dimakis A and Müller-Hoissen F 2007 With a Cole-Hopf transformation to solutions of the noncommutative KP hierarchy in terms of Wronski matrices to appear in J. Phys. A: Math. Theor.

[32] Dimakis A and Müller-Hoissen F 2006 Burgers and KP hierarchies: A functional representation approach to appear in Theor. Math. Phys. [nlin.SI/0601001]

[33] Falqui G, Magri F and Pedroni M 1998 Bihamiltonian geometry, Darboux coverings, and linearization of the KP hierarchy Commun. Math. Phys. 197 303-324 
[34] Guil F, Mañas M and Álvarez G 1994 The Hopf-Cole transformation and the KP equation Phys. Lett. A $19049-52$

[35] Dimakis A and Müller-Hoissen F 2006 From nonassociativity to solutions of the KP hierarchy Czech. J. Phys. $561123-1130$

[36] Dickey L A 2003 Soliton Equations and Hamiltonian Systems (Singapore: World Scientific)

[37] Gutt S and Rawnsley J 1999 Equivalence of star products on a symplectic manifold; an introduction to Deligne's Čech cohomology classes J. Geom. Phys. 29 347-392

[38] Nambu Y 1973 Generalized Hamiltonian dynamics Phys. Rev. D 7 2405-2412

[39] Takhtajan L 1994 On foundations of the generalized Nambu mechanics Commun. Math. Phys. 160 295-315

[40] Dito G, Flato M, Sternheimer D and Takhtajan L 1997 Deformation quantization and Nambu mechanics Commun. Math. Phys. 183 1-22

[41] Awata H, Li M, Minic D and Yoneya T 2001 On the quantization of Nambu brackets JHEP 02013

[42] Curtright T and Zachos C 2003 Classical and quantum Nambu mechanics Phys. Rev. D 68085001

[43] Hietarinta J 1997 Nambu tensors and commuting vector fields J. Phys. A: Math. Gen. 30 L27-L33

[44] Gessel I M 1984 Multipartite P-partitions and inner products of skew Schur functions Contemp. Math. 34 289-301

[45] Reutenauer C 1993 Free Lie Algebras (Oxford: Clarendon Press)

[46] Hazewinkel M 2000 Quasi-symmetric functions Formal Power Series and Algebraic Combinatorics ed D Krob, A A Mikhalev and A V Mikhalev (Berlin: Springer) pp 30-44

[47] Dorfmeister J, Neher E and Szmigielski J 1989 Automorphisms of Banach manifolds associated with the KP-equation Quart. J. Math. Oxford 40 161-195

[48] Macdonald I G 1995 Symmetric functions and Hall polynomials (Oxford: Oxford University Press)

[49] Olver P J and Sanders J A 2000 Transvectants, modular forms, and the Heisenberg algebra Adv. Math. 25 252-283

[50] Athorne C 2007 Applications of transvectants Preprint (Univ. of Glasgow) 